\title{
A Polyhedral Description of Kernels
}

\author{
Qin Chen $^{a}$ Xujin Chen ${ }^{b *}$ Wenan Zang ${ }^{c \dagger \ddagger}$ \\ a Department of Mathematics, China Jiliang University \\ Hangzhou 310018, China \\ $b$ Institute of Applied Mathematics, Chinese Academy of Sciences \\ Beijing 100190, China \\ c Department of Mathematics, The University of Hong Kong \\ Hong Kong, China
}

\begin{abstract}
Let $G$ be a digraph and let $\pi(G)$ be the linear system consisting of nonnegativity, stability, and domination inequalities. We call $G$ kernel ideal if $\pi(H)$ defines an integral polytope for each induced subgraph $H$ of $G$, and call $G$ kernel Mengerian if $\pi(H)$ is totally dual integral (TDI) for each induced subgraph $H$ of $G$. In this paper we show that a digraph is kernel ideal iff it is kernel Mengerian iff it contains none of three forbidden structures; our characterization yields a polynomial-time algorithm for the minimum weighted kernel problem on kernel ideal digraphs. We also prove that it is $N P$-hard to find a kernel of minimum size even in a planar bipartite digraph with maximum degree at most three.
\end{abstract}

MSC 2000 subject classification. Primary: 90C10, 90C27, 90C57.

OR/MS subject classification. Primary: Programming/graphs.

Key words. Digraph, kernel, polytope, algorithm, complexity.

\footnotetext{
* Supported in part by NSF of China under Grant 10771209 and by CAS Program for Cross and Cooperative Team of Science and Technology Innovation.

${ }^{\dagger}$ Supported in part by the Research Grants Council of Hong Kong.

${ }^{\ddagger}$ Corresponding author. E-mail: wzang@maths.hku.hk.
} 


\section{Introduction}

Digraphs considered in this paper contain no loops nor parallel arcs unless otherwise stated. Let $G=(V, A)$ be a digraph and let $U$ be a subset of $V$. We call $U$ a stable set of $G$ if no two vertices in $U$ are connected by an $\operatorname{arc}$ in $G$, call $U$ a dominating set of $G$ if for each vertex $v \notin U$, there is an arc from $v$ to $U$ in $G$, and call $U$ a kernel of $G$ if it is both stable and dominating in $G$. The study of kernels dates back to 1944 when von Neumann and Morgerstern [18] employed them to describe the winning positions in 2-person games; since then kernels have attracted tremendous research effort, and have been used as powerful tools for tackling many important problems arising in diverse research fields, such as logic, computational complexity, artificial intelligence, combinatorics, and coding theory. As shown by Chvátal [10], it is NP-complete in general to decide if a digraph has a kernel; this decision problem remains $N P$-complete even when restricted to planar digraphs with degree at most three (see Fraenkel [7]). So the focus of extensive research concerning kernels has been on special classes of digraphs.

Throughout this paper by a cycle (or a path) in a digraph we mean a directed one. We call a digraph $G$ even if it contains no odd cycles, and call $G$ bipartite if its underlying graph is bipartite. Von Neumann and Morgerstern [18] observed that every acyclic digraph contains a kernel, which was generalized by Richardson [15] (see Theorem 12.3.2 in [2]) as follows.

Theorem 1.1. [15] Every even digraph has a kernel.

Richardson [15] established this result by showing that

$$
\text { every strongly connected even digraph } H \text { is bipartite, }
$$

and thus each color class is a kernel of $H$. Applying (1.1) to a strongly connected component $H$ with no outgoing arcs, we can recursively find a kernel in an arbitrary even digraph $G$ in polynomial time.

Kernels are closely related to the so-called stable matchings. As demonstrated by Irving [13], the stable matching problem on a graph with strict preference orders can be solved in polynomial time. It follows that if a digraph $G$ is an orientation of a line graph and each clique of $G$ is acyclic, then there is a polynomial-time algorithm for finding a kernel in $G$, if any. For a digraph $G$ with a perfect underlying graph, Boros and Gurvich [4] proved that if in every clique the subgraph induced by one-way arcs is acyclic, then $G$ has a kernel; see Aharoni and Holzman [1] for a shorter proof based on Scarf's lemma and Király and Pap [12] for another shorter proof using Sperner's lemma and for an extension to $h$-perfect graphs. A more general setting where Sperner's lemma applies was studied by Edmonds, Gaubert and Gurvich [8]. We point out that, despite the existence, none of these proofs leads to a polynomial-time algorithm for finding a kernel. An open problem posed by Egerváry Research Group on Combinatorial Optimization (EGRES) is to find kernels in special classes of digraphs, including the aforementioned ones.

Problem 1.1. [6] In which classes of digraphs can we decide if a kernel exists and find one in polynomial time?

Given a digraph $G$ with an integral weight $w(v)$ on each vertex $v$, the minimum weighted kernel problem (MWKP) is to find a kernel in $G$ with minimum total weight. As remarked in the Egres Open [6] and confirmed by the following theorem, the MWKP is considerably more difficult than the existence problem (recall the above Richardson's theorem). 
Theorem 1.2. Given a planar bipartite digraph $G$ with maximum degree at most three and given a positive integer $k$, it is NP-complete to decide if $G$ has a kernel with at most $k$ vertices.

Motivated by the following Egres open problem, we study the MWKP using polyhedral and linear programming approaches in this paper.

Problem 1.2. [6] For which classes of digraphs can we explicitly give a linear description of the convex hull of kernels?

Let us introduce some notations and terminology before proceeding. As usual, we use $\mathbb{R}$ and $\mathbb{Z}$ to denote the sets of real numbers and integers, respectively. For any two sets $\Omega$ and $K$, where $\Omega$ is always a set of numbers and $K$ is always finite, we use $\Omega^{K}$ to denote the set of vectors $\boldsymbol{x}=(x(k): k \in K)$ whose coordinates are members of $\Omega$. Let $A \boldsymbol{x} \geq \boldsymbol{b}$ be a linear system and let $P$ denote the polyhedron $\{\boldsymbol{x}: A \boldsymbol{x} \geq \boldsymbol{b}\}$. We call $P$ integral if $P$ is the convex hull of all the integral vectors contained in it. It is well known that $P$ is integral if and only if the minimum in the LP-duality equation

$$
\min \left\{\boldsymbol{w}^{T} \boldsymbol{x}: A \boldsymbol{x} \geq \boldsymbol{b}\right\}=\max \left\{\boldsymbol{y}^{T} \boldsymbol{b}: \boldsymbol{y}^{T} A=\boldsymbol{w}^{T}, \boldsymbol{y} \geq \mathbf{0}\right\}
$$

has an integral optimal solution, for every integral vector $\boldsymbol{w}$ for which the optimum is finite. If, instead, the maximum in the equation enjoys this property, then the system $A \boldsymbol{x} \geq \boldsymbol{b}$ is called totally dual integral (TDI) [17]. The model of TDI systems plays a crucial role in combinatorial optimization, and serves as a general framework for establishing various min-max theorems because, as shown by Edmonds and Giles [5], total dual integrality implies primal integrality: if $A \boldsymbol{x} \geq \boldsymbol{b}$ is TDI and $\boldsymbol{b}$ is integral, then $P$ is integral.

Let $G=(V, A)$ be a digraph. For each $U \subseteq V$ (resp. $U \subseteq A$ ), we use $G \backslash U$ to denote the digraph obtained from $G$ by deleting all members of $U$, and set $G \backslash u=G \backslash U$ and $V \backslash U=V \backslash u$ (resp. $A \backslash U=A \backslash u$ ) if $U=\{u\}$. For each $v \in V$, we use $N_{G}^{+}(v)$ (resp. $N_{G}^{-}(v)$ ) to denote the set of all out-neighbors (resp. in-neighbors) of vertex $v$, and set $d_{G}^{+}(v)=\left|N_{G}^{+}(v)\right|$ (resp. $\left.d_{G}^{-}(v)=\left|N_{G}^{-}(v)\right|\right)$; we shall drop the subscript $G$ if there is no danger of confusion. Let $\pi(G)$ denote the following linear system:

$$
\begin{aligned}
0 \leq x(v) \leq 1 & & \forall v \in V, \\
x(u)+x(v) \leq 1 & & \forall(u, v) \in A, \\
x(v)+x\left(N_{G}^{+}(v)\right) \geq 1 & & \forall v \in V,
\end{aligned}
$$

where and throughout $x(U)=\sum_{u \in U} x(u)$ for any $U \subseteq V$. Let $\mathcal{C}$ be the collection of all cliques of $G$, and let $\sigma(G)$ be the linear system consisting of (1.2), (1.4), and the following inequalities:

$$
x(C) \leq 1 \quad \forall C \in \mathcal{C} .
$$

Observe that the upper bound in (1.2) is redundant unless $v$ is an isolated vertex, and that

the incidence vectors of kernels of $G$ are precisely integral solutions $\boldsymbol{x} \in \mathbb{Z}^{V}$ of $\pi(G)$.

The statement remains valid if we replace $\pi(G)$ by $\sigma(G)$. In the literature (1.2) - (1.5) are referred to as nonnegativity, stability, domination, and clique inequalities, respectively. The kernel polytope of $G$, denoted by $K(G)$, is the convex hull of incidence vectors of all kernels of $G$. It is clear that Problem 1.2 essentially asks for the defining system of $K(G)$.

The following is a reformulation of Rothblum's theorem [16] on stable matchings. 
Theorem 1.3. [16] Let $G$ be an orientation of the line graph of a bipartite graph in which every clique is acyclic. Then $K(G)$ is defined by $\sigma(G)$.

In [11], Király and Pap obtained the following strengthening of this result.

Theorem 1.4. [11] Let $G$ be an orientation of the line graph of a bipartite graph in which every clique is acyclic. Then $\sigma(G)$ is a TDI system.

While these two theorems concern themselves with the system $\sigma(G)$, the present paper aims to explore integrality properties enjoyed by $\pi(G)$. Let $F K(G)$ denote the set of all solutions $\boldsymbol{x} \in \mathbb{R}^{V}$ of $\pi(G)$. We call $F K(G)$ the fractional kernel polytope of $G$. Clearly, $K(G) \subseteq F K(G)$; however, equality need not hold in general. Thus a natural question to ask is the following.

Problem 1.3. How difficult is it to recognize all digraphs $G$ for which $K(G)=F K(G)$ (resp. $\pi(G)$ is $T D I)$ ?

It is worthwhile pointing out that, first, $K(G)=F K(G)$ iff $F K(G)$ is integral; second, if a digraph $G$ happens to enjoy this primal integrality (resp. total dual integrality), then this fact cannot be certified by exhibiting "forbidden" subgraphs of $G$, because every digraph $H$ is an induced subgraph of a digraph $G$ for which $K(G)=F K(G)$ (resp. $\pi(G)$ is TDI). To see this, add a new vertex $v$ to $H$ and $\operatorname{arcs}(u, v)$ for all vertices $u$ of $H$. Then the resulting digraph $G$ has a unique kernel $\{v\}$ and hence is as desired. Even in the absence of sinks, the integrality property $K(G)=F K(G)$ is still not closed under taking subgraphs or induced subgraphs: Let $G$ be the digraph depicted in Figure 1. Then $K(G)=F K(G)$ but neither $G \backslash v_{5}$ nor $G \backslash\left\{\left(v_{2}, v_{5}\right),\left(v_{5}, v_{6}\right)\right\}$ satisfies the corresponding equality. (To justify this, let $\boldsymbol{x}$ be an arbitrary vector in $F K(G)$, let $\theta$ be the value of $x\left(v_{4}\right)$, and let $\delta$ be the value of $x\left(v_{6}\right)$. Using (1.2) - (1.4), it can be shown that $\theta=\delta$, and $x\left(v_{i}\right)=\theta$ if $i$ is even and $1-\theta$ otherwise. Note that both $\left\{v_{1}, v_{3}, v_{5}\right\}$ and $\left\{v_{2}, v_{4}, v_{6}\right\}$ are kernels of $G$. Moreover, $G \backslash v_{5}$ is a so-called gear, whose fractional kernel polytope is not integral as we shall show in the main theorem.) So it is unlikely to have a characterization of all digraphs $G$ with $K(G)=F K(G)$ in terms of forbidden structures. Therefore, Problem 1.3 would be very challenging, if not intractable.

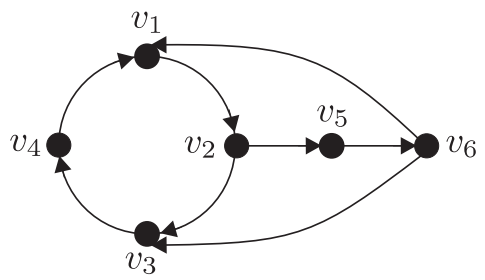

Figure 1: A digraph $G$ with $K(G)=K F(G)$ and $K\left(G \backslash v_{5}\right) \neq F K\left(G \backslash v_{5}\right)$.

A digraph $G$ is called kernel ideal if $K(H)=F K(H)$ for each induced subgraph $H$ of $G$, and called kernel Mengerian if $\pi(H)$ is a TDI system for each induced subgraph $H$ of $G$. By the aforementioned Edmonds-Giles theorem [5], every kernel Mengerian digraph is kernel ideal. The main purpose of this paper is to give a structural characterization of all kernel ideal and kernel Mengerian digraphs. 
We digress to define a few terms before presenting the main result. In this paper a path $P$ is a finite sequence $v_{0} v_{1} \ldots v_{k}$, such that $\left(v_{i}, v_{i+1}\right)$ is an arc for $1 \leq i \leq k-1$, and that $v_{0}, v_{1}, \ldots, v_{k}$ are distinct except possibly $v_{0}=v_{k}$ (in this case $P$ is a cycle). We follow the convention to call $P$ a $v_{0}-v_{k}$ path, and call $v_{0}$ the origin of $P$ and $v_{k}$ the terminus of $P$; both $v_{0}$ and $v_{k}$ are referred to as the ends of $P$. The length of $P$ is denoted by $|P|$. For any two vertices $v_{i}$ and $v_{j}$ on $P$ with $i<j$, we use $P\left[v_{i}, v_{j}\right]$ to denote the segment of $P$ from $v_{i}$ to $v_{j}$, and set $P\left(v_{i}, v_{j}\right]=P\left[v_{i}, v_{j}\right] \backslash v_{i}$, $P\left[v_{i}, v_{j}\right)=P\left[v_{i}, v_{j}\right] \backslash v_{j}$, and $P\left(v_{i}, v_{j}\right)=P\left[v_{i}, v_{j}\right] \backslash\left\{v_{i}, v_{j}\right\}$. For notational simplicity, we also write $u \in P$ for $u \in V(P)$. Given a directed cycle $C$ and two vertices $a, b$ on $C$, we use $C[a, b]$ to denote the segment of $C$ from $a$ to $b$.

A digraph $C$ is called a circuit if either its underlying graph is an undirected cycle, but $C$ itself is not a directed cycle, or $C$ is the digraph (on two vertices) formed by two parallel arcs. Notice that the number of sources equals the number of sinks in any circuit.

Let $C$ be a circuit, let $t_{1}, t_{2}, \ldots, t_{k}$ be all the sinks of $C$, and let $O_{1}, O_{2}, \ldots, O_{k}$ be $k$ vertexdisjoint cycles outside $C$. For each $i=1,2, \ldots, k$, we perform precisely one of the following three operations with respect to $t_{i}$ :

- identify $t_{i}$ with a vertex on $O_{i}$;

- add a directed path $P_{i}$ from $t_{i}$ to $O_{i}$;

- split $t_{i}$ into two vertices $t_{i}^{1}$ and $t_{i}^{2}$ (each of them is incident with one $\operatorname{arc}$ on $C$ ) and identify $t_{i}^{1}$ with a vertex on $O_{i}$ and $t_{i}^{2}$ with another vertex on $O_{i}$.

The resulting digraph is called a gear if it is even and contains no parallel arcs. Furthermore, $P_{i}$ 's are pairwise vertex-disjoint.

A ring is obtained from a directed cycle $C$ by adding an $s_{i}$ - $t_{i}$ path $P_{i}$ for $i=1,2$, such that

- $s_{1}, t_{1}, s_{2}, t_{2}$ occur on $C$ in order when we traverse $C$ in its direction from $s_{1}$;

- $s_{i} \neq t_{i}$ (but possibly $s_{i}=t_{3-i}$ ) for $i=1,2$;

- $P_{1}\left(s_{1}, t_{1}\right), P_{2}\left(s_{2}, t_{2}\right)$, and $C$ are pairwise vertex-disjoint;

- $P_{1} \cup P_{2} \cup C$ is even and contains no parallel arcs; and

- $\left|C\left[s_{1}, s_{2}\right]\right|$ is odd.

Now we are ready to state the main theorem of this paper.

Theorem 1.5. For a digraph $G$, the following statements are equivalent:

(i) $G$ contains no subgraph isomorphic to an odd cycle, a gear, or a ring;

(ii) $G$ is kernel ideal; and

(iii) $G$ is kernel Mengerian.

To interpret statements (ii) and (iii) in the preceding theorem, let $\mathbb{P}(G, \boldsymbol{w})$ stand for the linear program

$$
\begin{array}{ll}
\text { Minimize } & \boldsymbol{w}^{T} \boldsymbol{x} \\
\text { subject to } & \boldsymbol{x} \in F K(G),
\end{array}
$$

and let $\mathbb{D}(G, \boldsymbol{w})$ denote its dual. Note that if $G$ contains no isolated vertex, then (1.2) can be replaced by $x(v) \geq 0$ for all $v \in V$ in the definition of $F K(G)$. Thus $\mathbb{D}(G, \boldsymbol{w})$ can be simplified 


$$
\begin{aligned}
& \text { Maximize } \quad-y(A)+z(V) \\
& \text { subject to } \quad-\sum_{v \in e} y(e)+z(v)+z\left(N_{G}^{-}(v)\right) \leq w(v) \quad \forall v \in V, \\
& y(e) \geq 0 \quad \forall e \in A, \\
& z(v) \geq 0 \quad \forall v \in V,
\end{aligned}
$$

where by $v \in e$ we mean $v$ is an end of $\operatorname{arc} e$. Using these notations, we see that $G$ is kernel ideal if and only if $\mathbb{P}(H, \boldsymbol{w})$ has an integral optimal solution for any induced subgraph $H$ of $G$ and any $\boldsymbol{w} \in \mathbb{Z}^{V(H)}$, and that $G$ is kernel Mengerian if and only if both $\mathbb{P}(H, \boldsymbol{w})$ and $\mathbb{D}(H, \boldsymbol{w})$ have integral optimal solutions for any induced subgraph $H$ of $G$ and any $\boldsymbol{w} \in \mathbb{Z}^{V(H)}$ (and hence a combinatorial min-max relation follows).

Theorem 1.5 gives a structural description of all kernel ideal and kernel Mengerian digraphs in terms of forbidden structures. We point out that our characterization is actually a counterpart in the polyhedral case of the aforementioned Richardson's theorem (see Theorem 1.1), which can be rephrased as: Let $G$ be a digraph. Then each subgraph of $G$ has a kernel if and only if $G$ contains no odd cycle. Interestingly, despite extensive research, a good characterization of all digraphs $G$ such that each induced subgraph of $G$ contains a kernel has yet to be found (see, for instance, $[6,9]$ ). It is because kernels are usually not so well-behaved that these combinatorial objects are still surrounded by mystery. We close this section with two more open problems, which are intimately related to Theorem 1.5.

Problem 1.4. Characterize all digraphs $G$ such that $\sigma(H)$ defines an integral polytope for each induced subgraph $H$ of $G$.

Problem 1.5. Characterize all digraphs $G$ such that $\sigma(H)$ is TDI for each induced subgraph $H$ of $G$.

\section{Complexity}

Recall that every even digraph has a kernel; Richardson's proof [15] actually yields a polynomialtime algorithm for finding such a kernel. Let us now show that the MWKP is NP-hard even for some very special class of bipartite digraphs.

Proof of Theorem 1.2. Obviously, the kernel problem in our consideration is in NP. To prove the assertion, it suffices to reduce the planar 3-SATISFIABILITY problem (P3SAT) $[14,10]$ to this problem. Let $U=\left\{u_{1}, u_{2}, \ldots, u_{n}\right\}$ be the set of variables, let $\mathcal{C}=\left\{C_{1}, C_{2}, \ldots, C_{m}\right\}$ be the set of clauses in an arbitrary instance of the P3SAT in $C N F$, and let $H$ be the bipartite graph with vertex set $U \cup \mathcal{C}$ such that $u_{i} C_{j}$ is an edge of $H$ if and only if $u_{i} \in C_{j}$ or $\bar{u}_{i} \in C_{j}$ for $1 \leq i \leq n$ and $1 \leq j \leq m$. (Without loss of generality, we assume that no clause in $\mathcal{C}$ contains both $u_{i}$ and $\bar{u}_{i}$ for any $i$.) By definition, $H$ admits a plane embedding. For convenience, we view $H$ as a plane graph hereafter, and use $e_{i}^{1}, e_{i}^{2}, \ldots, e_{i}^{d_{i}}$ to denote all the edges incident with $u_{i}$ in clockwise order in $H$ for each $i$. Note that $d_{i}$ equals the total number of occurrences of $u_{i}$ and $\bar{u}_{i}$ in $\mathcal{C}$. 
Our objective is to construct a planar bipartite digraph $G$ with maximum degree at most three so that there exists a kernel in $G$ with at most $18 m$ vertices if and only if $\mathcal{C}$ is satisfiable. We can obtain the desired $G$ from $H$ by blowing up its vertices as described below:

(1) Replace each vertex $u_{i}$ in $H$ by a truth-setting component $T_{i}$ with vertex set $\cup_{t=1}^{d_{i}}\left(\left\{a_{i, t}^{1}, a_{i, t}^{2}\right.\right.$, $\left.\left.\ldots, a_{i, t}^{8}\right\} \cup\left\{b_{i, t}^{1}, b_{i, t}^{2}, b_{i, t}^{3}, b_{i, t}^{4}\right\}\right)$, such that $T_{i}$ contains four (directed) paths $a_{i, t}^{3} a_{i, t}^{2} a_{i, t}^{1}, a_{i, t}^{3} a_{i, t}^{4} a_{i, t}^{5}$, $a_{i, t}^{7} a_{i, t}^{6} a_{i, t}^{5}, a_{i, t}^{7} a_{i, t}^{8} a_{i, t+1}^{1}$, two (directed) cycles $b_{i, t}^{k} b_{i, t}^{k+1} b_{i, t}^{k}$ for $k=1,3$, and two additional $\operatorname{arcs}\left(a_{i, t}^{1}, b_{i, t}^{1}\right)$ and $\left(a_{i, t}^{5}, b_{i, t}^{3}\right)$, for each $t=1,2, \ldots, d_{i}$, where $d_{i}$ is the degree of $u_{i}$ in $H$ and $a_{i, d_{i}+1}^{1}=a_{i, 1}^{1}$. (So $T_{i}$ has $12 d_{i}$ vertices and $14 d_{i}$ arcs in total; see Figure 2 . Note that $\cup_{t=1}^{d_{i}}\left\{a_{i, t}^{1}, a_{i, t}^{2}, \ldots, a_{i, t}^{8}\right\}$ induces an undirected cycle in the underlying graph of $T_{i}$.)

(2) Replace each vertex $C_{j}$ in $H$ by a satisfaction-testing component $S_{j}$ with vertex set $\left\{x_{j}, y_{j}^{1}, y_{j}^{2}, y_{j}^{3}, z_{j}^{1}, z_{j}^{2}, z_{j}^{3}\right\}$, such that $x_{j} y_{j}^{k} z_{j}^{k}$ is a path for $k=1,2,3$. (So $S_{j}$ has 7 vertices and 6 arcs in total; see Figure 2.)

(3) For each edge $u_{i} C_{j}$ in $H$, we have $u_{i} \in C_{j}$ or $\bar{u}_{i} \in C_{j}$. Let $r_{j}^{1}, r_{j}^{2}$, and $r_{j}^{3}$ denote the three literals in $C_{j}$ throughout, and suppose $u_{i} C_{j}=e_{i}^{t}$ for some $t$ with $1 \leq t \leq d_{i}$. If $u_{i}=r_{j}^{k}$, then add an arc from $z_{j}^{k}$ to $a_{i, t}^{2}$; if $\bar{u}_{i}=r_{j}^{k}$, then add an arc from $z_{j}^{k}$ to $a_{i, t}^{6}$.

The construction of $G$ is completed. It is easy to see that this construction can be accomplished in polynomial time, $G$ has $43 m$ vertices and $51 m$ edges (because $\sum_{i=1}^{n} d_{i}=3 m$ ), and $G$ is a planar bipartite digraph with maximum degree at most three.

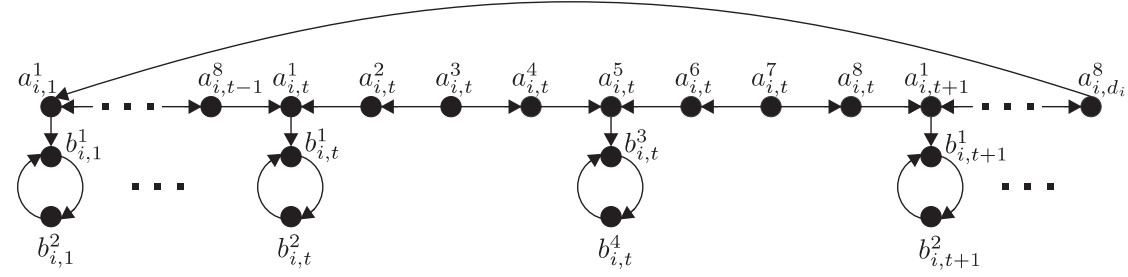

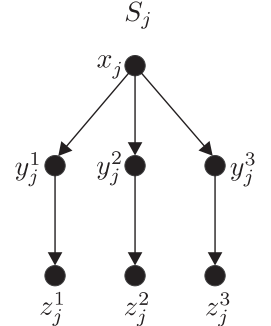

Figure 2: The truth-setting component $T_{i}$ and satisfaction-testing component $S_{j}$.

Let us show that $G$ has a kernel with at most $18 \mathrm{~m}$ vertices if and only if $\mathcal{C}$ is satisfiable.

Sufficiency. Suppose that $\tau: U \rightarrow\{$ true, false $\}$ is a satisfying truth assignment for $\mathcal{C}$. We construct a vertex subset $K$ of $G$ as follows. For each variable $u_{i}$, if $\tau\left(u_{i}\right)=$ true, then $\cup_{i=1}^{d_{i}}\left(\left\{a_{i, t}^{2}, a_{i, t}^{5}, a_{i, t}^{8}\right\} \cup\left\{b_{i, t}^{1}, b_{i, t}^{4}\right\}\right) \subseteq K$; if $\tau\left(u_{i}\right)=$ false, then $\cup_{i=1}^{d_{i}}\left(\left\{a_{i, t}^{1}, a_{i, t}^{4}, a_{i, t}^{6}\right\} \cup\left\{b_{i, t}^{2}, b_{i, t}^{3}\right\}\right) \subseteq K$. For each clause $C_{j} \in \mathcal{C}$ and $k=1,2,3$, if $\tau\left(r_{j}^{k}\right)=$ true, then $y_{j}^{k} \in K$; if $\tau\left(r_{j}^{k}\right)=$ false, then $z_{j}^{k} \in K$. This completes the construction of $K$. Clearly, $|K|=18 m$ (recall that $\sum_{i=1}^{n} d_{i}=3 m$ ), $K$ is a stable set, and $K \cap V\left(T_{i}\right)$ is a kernel of $T_{i}$ for $1 \leq i \leq n$. Since for each $j=1,2, \ldots, m$, there exists at least one $k$ with $\tau\left(r_{j}^{k}\right)=$ true and thus $y_{j}^{k} \in K$, and since $\left(x_{j}, y_{j}^{k}\right)$ is an arc, we deduce that $K$ is a kernel of $G$.

Necessity. Suppose $G$ has a kernel $K$ with at most $18 m$ vertices. Then

(4) $\left|K \cap\left\{b_{i, t}^{k}, b_{i, t}^{k+1}\right\}\right|=1$ for $1 \leq i \leq n, 1 \leq t \leq d_{i}$, and $k=1,3$.

From the structure of $G$, it can be seen that

(5) for each $j=1,2, \ldots, m$, we have $\left|K \cap V\left(S_{j}\right)\right| \geq 3$ and equality holds only when $y_{j}^{h_{j}} \in K$ for at least one subscript $h_{j}$ with $1 \leq h_{j} \leq 3$. 
We propose to show that

(6) for each $i=1,2, \ldots, n$, we have $\left|K \cap V\left(T_{i}\right)\right| \geq 5 d_{i}$ and equality holds only when $K \cap V\left(T_{i}\right)=$ $\cup_{t=1}^{d_{i}}\left(\left\{a_{i, t}^{2}, a_{i, t}^{5}, a_{i, t}^{8}\right\} \cup\left\{b_{i, t}^{1}, b_{i, t}^{4}\right\}\right)$ or $K \cap V\left(T_{i}\right)=\cup_{t=1}^{d_{i}}\left(\left\{a_{i, t}^{1}, a_{i, t}^{4}, a_{i, t}^{6}\right\} \cup\left\{b_{i, t}^{2}, b_{i, t}^{3}\right\}\right)$.

To this end, set $K_{i, t}=K \cap\left\{a_{i, t}^{1}, a_{i, t}^{2}, \ldots, a_{i, t}^{8}\right\}$. Observe that

(7) $\left|K_{i, t}\right| \geq 3$ for each $t=1,2, \ldots, d_{i}$, because $K \cap V\left(T_{i}\right)$ is a kernel of $T_{i}$.

Combining (4) and (7), we obtain

(8) $\left|K \cap V\left(T_{i}\right)\right|=2 d_{i}+\sum_{t=1}^{d_{i}}\left|K_{i, t}\right| \geq 5 d_{i}$.

From this inequality and (5), it follows that $18 m \geq|K| \geq \sum_{i=1}^{n} 5 d_{i}+3 m=18 m$. Therefore

(9) $\left|K \cap V\left(T_{i}\right)\right|=5 d_{i}$ for $1 \leq i \leq n$, and $\left|K \cap V\left(S_{j}\right)\right|=3$ for $1 \leq j \leq m$.

The first equality in (9) and (8) in turn imply that

(10) $\left|K_{i, t}\right|=3$ for each $t=1,2, \ldots, d_{i}$.

Let us consider the case when $a_{i, s}^{1} \in K_{i, s}$ for some $s$ with $1 \leq s \leq d_{i}$. Since $K \cap V\left(T_{i}\right)$ is a kernel of $T_{i}$ and $K_{i, s} \subseteq K \cap V\left(T_{i}\right)$, it is a routine matter to check, using (10), that $K_{i, s}=$ $\left\{a_{i, s}^{1}, a_{i, s}^{4}, a_{i, s}^{6}\right\}$. In view of the vertex $a_{i, s}^{8}$, we obtain $a_{i, s+1}^{1} \in K_{i, s+1}$. Applying induction on the subscript $t$ from $s$, we see that $K_{i, t}=\left\{a_{i, t}^{1}, a_{i, t}^{4}, a_{i, t}^{6}\right\}$ for all $t=1,2, \ldots, d_{i}$. Hence $K \cap V\left(T_{i}\right)=$ $\cup_{t=1}^{d_{i}}\left(\left\{a_{i, t}^{1}, a_{i, t}^{4}, a_{i, t}^{6}\right\} \cup\left\{b_{i, t}^{2}, b_{i, t}^{3}\right\}\right)$.

So we assume that $a_{i, t}^{1} \notin K_{i, t}$ for all $t$ with $1 \leq t \leq d_{i}$. From (10), it follows instantly that $K_{i, t}=\left\{a_{i, t}^{2}, a_{i, t}^{5}, a_{i, t}^{8}\right\}$ for each $t$. Hence $K \cap V\left(T_{i}\right)=\cup_{t=1}^{d_{i}}\left(\left\{a_{i, t}^{2}, a_{i, t}^{5}, a_{i, t}^{8}\right\} \cup\left\{b_{i, t}^{1}, b_{i, t}^{4}\right\}\right)$. Therefore (6) holds.

Let us now define a truth assignment $\tau: U \rightarrow\{$ true, false $\}$ by setting $\tau\left(u_{i}\right)=$ true if $K \cap V\left(T_{i}\right)=\cup_{t=1}^{d_{i}}\left(\left\{a_{i, t}^{2}, a_{i, t}^{5}, a_{i, t}^{8}\right\} \cup\left\{b_{i, t}^{1}, b_{i, t}^{4}\right\}\right)$ and setting $\tau\left(u_{i}\right)=$ false if $K \cap V\left(T_{i}\right)=$ $\cup_{t=1}^{d_{i}}\left(\left\{a_{i, t}^{1}, a_{i, t}^{4}, a_{i, t}^{6}\right\} \cup\left\{b_{i, t}^{2}, b_{i, t}^{3}\right\}\right)$, for $i=1,2 \ldots, n$. It remains to verify that each clause $C_{j}$ is satisfied by $\tau$. Indeed, by (9), the subscript $h_{j}$ as specified in (5) exists for each $j=1,2, \ldots, m$, which implies that $G$ has an arc from $z_{j}^{h_{j}}$ to $K \cap V\left(T_{i}\right)$ for some $i$. From the definition of $\tau$, we deduce that $\tau\left(r_{j}^{h_{j}}\right)=$ true. Therefore $C_{j}$ is satisfied by $\tau$ for all $j$, completing the proof.

\section{Obstructions}

In this section we show that odd cycles, gears, and rings are all obstructions for a digraph to be kernel ideal.

Lemma 3.1. Let $G$ be a digraph that contains an odd cycle as a subgraph. Then $G$ is not kernel ideal.

Proof. Let $C$ be an odd cycle in $G$ and let $\hat{C}=(V, A)$ be the subgraph of $G$ induced by all vertices in $C$. Consider $\boldsymbol{w} \in \mathbb{Z}^{V}$ and $\boldsymbol{a} \in \mathbb{Q}^{V}$ such that $w(v)=-1$ and $a(v)=1 / 2$ for each $v \in V$. Clearly $\boldsymbol{a} \in F K(\hat{C})$, so $F K(\hat{C}) \neq \emptyset$. Since $C$ is an odd cycle, each kernel of $\hat{C}$ (if any) has size at most $(|V|-1) / 2$. Thus $\boldsymbol{w}^{T} \boldsymbol{a}=-|V| / 2<-(|V|-1) / 2 \leq \boldsymbol{w}^{T} \boldsymbol{x}$ for any incidence vector $\boldsymbol{x}$ of a kernel in $\hat{C}$. From (1.6) we see that $F K(\hat{C}) \neq K(\hat{C})$, and hence $G$ is not kernel ideal.

The remainder of this section is devoted to establishing the following statement. 
Theorem 3.2. Let $G$ be a digraph that contains a gear or a ring as a subgraph. Then $G$ is not kernel ideal.

We break the proof into a series of observations. Recall the definition, a gear $H$ is an even digraph (with no parallel arcs) obtained from the vertex-disjoint union of a circuit $C$ and $k$ cycles $O_{1}, O_{2}, \ldots, O_{k}$ by performing precisely one of the following three operations with respect to each sink $t_{i}$ of $C$ for $1 \leq i \leq k$ :

- identify $t_{i}$ with a vertex on $O_{i}$;

- add a directed path $P_{i}$ from $t_{i}$ to $O_{i}$;

- split $t_{i}$ into two vertices $t_{i}^{1}$ and $t_{i}^{2}$ (each of them is adjacent with one arc on $C$ ) and identify $t_{i}^{1}$ with a vertex on $O_{i}$ and $t_{i}^{2}$ with another vertex on $O_{i}$.

Let $s_{1}, s_{2}, \ldots, s_{k}$ be all the sources of $C$, which we call the distinguished vertices of $H$. Renaming the subscripts if necessary, we assume that $s_{1}, t_{1}, s_{2}, t_{2}, \ldots, s_{k}, t_{k}$ occur on $C$ in order if we traverse $C$ from $s_{1}$ in the clockwise direction.

Lemma 3.3. Let $H^{\prime}=(V, A)$ be a digraph that contains a gear $H$ as a spanning subgraph. If $d_{H^{\prime}}^{+}\left(s_{1}\right)=2$ (see the above description), then $H^{\prime}$ is not kernel ideal.

Proof. Let $\Lambda$ be the set of all subscripts $i$ such that the above third operation has been applied with respect to $t_{i}$ in the construction of $H$, and let $Q_{i}$ be the segment of $O_{i}$ from $t_{i}^{1}$ to $t_{i}^{2}$ for each $i \in \Lambda$. Then the arcs on $C$ and on $Q_{i}$ for all $i \in \Lambda$ form a circuit in $H$, denoted by $D$. Depending on the parity of $|V(D)|$, we consider two cases.

Case 1. $|V(D)|$ is odd.

In this case, define $\boldsymbol{w} \in \mathbb{Z}^{V}$ and $\boldsymbol{a} \in \mathbb{R}^{V}$ by

- $w(v)=-1$ if $v \in V(D)$ and $w(v)=0$ otherwise;

- $a(v)=1 / 2$ for each $v \in V$.

Clearly, $\boldsymbol{a} \in F K\left(H^{\prime}\right)$. Since $|V(D)|$ is odd, each kernel of $H^{\prime}$ (if any) contains at most $(\mid V(D \mid-$ $1) / 2$ vertices from $D$. Thus $\boldsymbol{w}^{T} \boldsymbol{a}=-|V(D)| / 2<-(|V(D)|-1) / 2 \leq \boldsymbol{w}^{T} \boldsymbol{x}$ for any incidence vector $\boldsymbol{x}$ of a kernel in $H^{\prime}$. From (1.6) we see that $F K\left(H^{\prime}\right) \neq K\left(H^{\prime}\right)$, and hence $H^{\prime}$ is not kernel ideal.

Case 2. $|V(D)|$ is even.

In this case, let $u_{1}$ and $u_{2}$ be the two out-neighbors of $s_{1}$ on $D$. Define $\boldsymbol{w} \in \mathbb{Z}^{V}$ and $\boldsymbol{a} \in \mathbb{R}^{V}$ by

- $w\left(s_{1}\right)=1, w\left(u_{i}\right)=0$ for $i=1,2, w(v)=-1$ if $v \in V(D) \backslash\left\{s_{1}, u_{1}, u_{2}\right\}$, and $w(v)=0$ if $v \notin V(D)$;

- $a\left(s_{1}\right)=0$ and $a(v)=1 / 2$ for each $v \in V \backslash s_{1}$;

Clearly, $\boldsymbol{a} \in F K\left(H^{\prime}\right)$. Let $\boldsymbol{x}$ be the incidence vector of an arbitrary kernel in $H^{\prime}$ (if any). If $x\left(u_{1}\right)$ or $x\left(u_{2}\right)$ is 1 , then $x\left(V(D) \backslash\left\{s_{1}, u_{1}, u_{2}\right\}\right) \leq(|V(D)|-4) / 2$ by the stability of the kernel; if $x\left(u_{1}\right)=x\left(u_{2}\right)=0$, then $x\left(s_{1}\right)=1\left(\right.$ as $\left.d_{H^{\prime}}^{+}\left(s_{1}\right)=2\right)$ and $x\left(V(D) \backslash\left\{s_{1}, u_{1}, u_{2}\right\}\right) \leq(|V(D)|-2) / 2$. In either case, we have $\boldsymbol{w}^{T} \boldsymbol{a}=-(|V(D)|-3) / 2<-(|V(D)|-4) / 2 \leq \boldsymbol{w}^{T} \boldsymbol{x}$, which implies that $F K\left(H^{\prime}\right) \neq K\left(H^{\prime}\right)$, and hence $H^{\prime}$ is not kernel ideal.

Let $H$ be a digraph obtained from a directed even cycle $C$ by adding a new vertex $v$ and some arcs between $v$ and $C$ so that no parallel arc is created. We call $H$ a wheel if there are at 
least three $\operatorname{arcs}$ from $v$ to $C$. Note that there might be arcs from $C$ to $v$ in $H$. We call $v$ the $h u b$ and $C$ the rim of $H$.

Lemma 3.4. Let $H=(V, A)$ be a wheel (see the above description). Then $H$ is not kernel ideal.

Proof. Let $C=u_{1} u_{2} \ldots u_{2 n} u_{1}$, and let $u_{i_{1}}, u_{i_{2}}, \ldots, u_{i_{k}}$ be all the out-neighbors of $v$, where $1 \leq i_{1}<i_{2}<\ldots<i_{k} \leq 2 n$. We proceed by considering two cases.

Case 1. $\left|i_{j+1}-i_{j}\right|$ is odd for some $j$ with $1 \leq j \leq k-1$.

In this case, let $D$ be the circuit formed by $C\left[u_{i_{j}}, u_{i_{j+1}}\right]$ and two $\operatorname{arcs}\left(v, u_{i_{j}}\right)$ and $\left(v, u_{i_{j+1}}\right)$. We define $\boldsymbol{w} \in \mathbb{Z}^{V}$ and $\boldsymbol{a} \in \mathbb{R}^{V}$ as follows:

- $w(u)=-1$ if $u \in V(D)$ and $w(u)=0$ otherwise;

- $a(u)=1 / 2$ for each $u \in V$.

Clearly, $\boldsymbol{a} \in F K(H)$. Since $|V(D)|$ is odd, each kernel of $H$ (if any) contains at most $(|V(D)|-$ $1) / 2$ vertices from $D$. Thus $\boldsymbol{w}^{T} \boldsymbol{a}=-|V(D)| / 2<-(|V(D)|-1) / 2 \leq \boldsymbol{w}^{T} \boldsymbol{x}$ for any incidence vector $\boldsymbol{x}$ of a kernel in $H$. So $F K(H) \neq K(H)$, and hence $H$ is not kernel ideal.

Case 2. $\left|i_{j+1}-i_{j}\right|$ is even for each $j$ with $1 \leq j \leq k-1$.

In this case, we may assume that $i_{j}$ is odd for each $j$ (renaming subscripts if necessary). Define $\boldsymbol{w} \in \mathbb{Z}^{V}$ and $\boldsymbol{a} \in \mathbb{R}^{V}$ by

- $w(v)=k, w\left(u_{i_{j}}\right)=0$ for $1 \leq j \leq k$, and $w(u)=-1$ if $u \in V(C) \backslash N_{H}^{+}(v)$; and

- $a(v)=0$ and $a(u)=1 / 2$ for each $u \in V \backslash v$.

Clearly, $\boldsymbol{a} \in F K(H)$. Let $\boldsymbol{x}$ be the incidence vector of an arbitrary kernel $U$ in $H$. If $x\left(u_{i_{j}}\right)=1$ for some $j$ with $1 \leq j \leq k$, then $U=\left\{u_{1}, u_{3}, \ldots, u_{2 n-1}\right\}$ by the hypothesis of the present case. So $\boldsymbol{w}^{T} \boldsymbol{x}=-n+k$. If $x\left(u_{i_{j}}\right)=0$ for each $j$ with $1 \leq j \leq k$, then $v \in U$ and $U \cap V(C) \subseteq\left\{u_{2}, v_{4}, \ldots, u_{2 n}\right\}$. So $\boldsymbol{w}^{T} \boldsymbol{x} \geq-n+k$. In either case, we have $\boldsymbol{w}^{T} \boldsymbol{x} \geq-n+k$. Hence $\boldsymbol{w}^{T} \boldsymbol{a}=-(2 n-k) / 2=-n+k / 2<\boldsymbol{w}^{T} \boldsymbol{x}$, which implies that $F K(H) \neq K(H)$, and hence $H$ is not kernel ideal.

Recall the definition, an odd ring $H$ is obtained from a directed cycle $C$ by adding an $s_{i}$ - $t_{i}$ path $P_{i}$ for $i=1,2$, such that

- $s_{1}, t_{1}, s_{2}, t_{2}$ occur on $C$ in order when we traverse $C$ in its direction from $s_{1}$;

- $s_{i} \neq t_{i}$ (but possibly $s_{i}=t_{3-i}$ ) for $i=1,2$;

- $P_{1}\left(s_{1}, t_{1}\right), P_{2}\left(s_{2}, t_{2}\right)$, and $C$ are pairwise vertex-disjoint;

- $P_{1} \cup P_{2} \cup C$ is even and contains no parallel arcs; and

- $\left|C\left[s_{1}, s_{2}\right]\right|$ is odd.

Lemma 3.5. Let $H=(V, A)$ be a ring. Then $H$ is not kernel ideal.

Proof. Since $H$ contains no odd cycles, $\left|C\left[s_{i}, t_{i}\right]\right|$ and $\left|P_{i}\right|$ have the same parity for $i=1,2$. So there is a one-to-one correspondence between the kernels of $H$ and those of $C$, and hence $H$ has precisely two kernels $X_{1}$ and $X_{2}$ which form the unique bipartition of $H$, where $s_{i} \in X_{i}$ for $i=1,2$. Let $\boldsymbol{x} \in \mathbb{R}^{V}$ be defined as follows:

- $x(v)=1 / 2$ for any vertex $v$ outside $C\left(s_{1}, s_{2}\right] \cup P_{1}\left(s_{1}, t_{1}\right]$;

- $x(v)=3 / 4$ for any vertex $v$ in $V\left(C\left(s_{1}, s_{2}\right] \cup P_{1}\left(s_{1}, t_{1}\right]\right) \cap X_{1}$;

- $x(v)=1 / 4$ for any vertex $v$ in $V\left(C\left(s_{1}, s_{2}\right] \cup P_{1}\left(s_{1}, t_{1}\right]\right) \cap X_{2}$. 
Clearly, $\boldsymbol{x} \in F K(H)$; however, it cannot be expressed a convex combination of the incidence vectors of $X_{1}$ and $X_{2}$, which implies that $F K(H) \neq K(H)$, so $H$ is not kernel ideal.

Lemma 3.6. Let $H^{\prime}=\left(V, A^{\prime}\right)$ be a digraph obtained from a ring $H=(V, A)$ by adding an arc that is not parallel to any arc in $H$. Then $H^{\prime}$ contains an odd cycle, or a gear, or a ring with at most $|V|-1$ vertices.

Proof. We assume that $H^{\prime}$ contains no odd cycle, otherwise we are done. Since $H$ is strongly connected, so is $H^{\prime}$ and hence it is bipartite by (1.1).

Recall the definition, $H$ is obtained from a cycle $C$ by adding an $s_{i}-t_{i}$ path $P_{i}$ for $i=1,2$ with the properties as described above the preceding lemma. Set $P_{3}=C\left[s_{1}, t_{1}\right]$ and $P_{4}=C\left[s_{2}, t_{2}\right]$. Obviously, $P_{1}$ and $P_{3}$ (similarly, $P_{2}$ and $P_{4}$ ) are in the same position in $H$. Let $e=(u, v)$ be the arc in $A^{\prime} \backslash A$. There are now five cases, corresponding to possible locations of $u$ and $v$.

Case 1. $u \in C\left[t_{1}, s_{2}\right]$ and $v \in C\left[t_{2}, s_{1}\right]$.

If $u=s_{2}$, let $i$ be a subscript in $\{2,4\}$ for which $P_{i}\left(s_{2}, t_{2}\right)$ contains at least one vertex, and let $\Sigma$ be obtained from $H^{\prime}$ by deleting all vertices in $P_{i}\left(s_{2}, t_{2}\right)$, then $\Sigma$ is a ring with at most $|V|-1$ vertices. If $u \neq s_{2}$, then $C\left[s_{2}, u\right] \cup P_{2} \cup\{(u, v)\}$ is a gear.

Case 2. $\{u, v\} \subseteq P_{1}$.

If $v \in P_{1}\left(u, t_{1}\right]$, let $\Sigma$ be obtained from $H$ by replacing $P_{1}[u, v]$ with $(u, v)$, then $\Sigma$ is a ring with at most $|V|-1$ vertices. So we suppose $v \in P_{1}\left[s_{1}, u\right)$. Furthermore, $\{u, v\} \neq\left\{t_{1}, s_{1}\right\}$, for otherwise Case 1 occurs. Let $\Sigma$ be obtained from $H^{\prime}$ by deleting all vertices in $P_{1}\left(u, t_{1}\right) \cup C\left(s_{1}, s_{2}\right)$ if $u \neq t_{1}$, and let $\Sigma$ be $P_{1} \cup P_{3} \cup\{(u, v)\}$ if $u=t_{1}$. Then $\Sigma$ is a gear in either subcase.

Case 3. $u \in P_{1}$ and $v \in P_{3}$.

In this case we may assume that $u \notin\left\{s_{1}, t_{1}\right\}$, for otherwise Case 2 occurs (with $P_{3}$ in place of $\left.P_{1}\right)$. Thus $C \cup P_{1}\left[u, t_{1}\right] \cup\{(u, v)\}$ is a gear.

Case 4. $u \in P_{1}\left(s_{1}, t_{1}\right)$ and $v \in P_{2}$.

Let $C^{\prime}$ be obtained from $C$ by replacing $P_{4}$ with $P_{2}$. Then $C^{\prime} \cup P_{1}\left[u, t_{1}\right] \cup\{(u, v)\}$ is a gear.

Case 5. $\{u, v\} \subseteq C\left[t_{1}, s_{2}\right]$.

If $v \in C\left(u, s_{2}\right]$, let $\Sigma$ be obtained from $H$ by replacing $C[u, v]$ with $(u, v)$, then $\Sigma$ is a ring with at most $|V|-1$ vertices. If $v \in C\left[t_{1}, u\right)$, then $P_{1} \cup C\left[s_{1}, u\right] \cup\{(u, v)\}$ is a gear.

Each of the remaining cases is a mirror image of one case listed above.

Proof of Theorem 3.2. Let $H$ be a gear or a ring in $G$ with the smallest number of vertices, and let $\hat{H}$ be the subgraph of $G$ induced by all vertices in $H$. We may assume that $\hat{H}$ is kernel ideal, otherwise we are done. By Lemma 3.5, $\hat{H}$ is not a ring. Thus Lemma 3.6 and Lemma 3.1 allow us to further assume that $H$ is a gear. Recall the structural description of $H$ above Lemma 3.3; using this lemma, we obtain $d_{\hat{H}}^{+}\left(s_{1}\right) \geq 3$. So $\hat{H}$ has an arc $\left(s_{1}, v\right)$ outside the circuit $C$. Depending on the structure of $H$, we distinguish between two cases.

Case 1. $H$ has precisely one distinguished vertex.

By assumption, $H \cup\left\{\left(s_{1}, v\right)\right\}$ contains no gear with fewer vertices than $H$. Consequently, $H$ is obtained from the cycle $O_{1}$ by adding two $\operatorname{arcs}\left(s_{1}, t_{1}^{1}\right)$ and $\left(s_{1}, t_{1}^{2}\right)$. As $\hat{H}$ is not a wheel with hub $s_{1}$ and rim $O_{1}$ by Lemma 3.4, there exists an $\operatorname{arc}(p, q)$ outside $O_{1}$ yet connecting two 
vertices on $O_{1}$. For convenience, set $t_{1}^{3}=v$. Swapping $t_{1}^{1}$ and $t_{1}^{2}$ if necessary, we assume that $t_{1}^{1}, t_{1}^{2}, t_{1}^{3}$ occur on $O_{1}$ in order if we traverse $O_{1}$ from $t_{1}^{1}$ in the clockwise direction.

Consider the subcase when $\{p, q\} \subseteq O_{1}\left[t_{1}^{1}, t_{1}^{2}\right]$. Let $\Sigma$ be obtained from $H$ by replacing $O_{1}[p, q]$ with the $\operatorname{arc}(p, q)$ if $q \in O_{1}\left(p, t_{1}^{2}\right]$, let $\Sigma$ be $O_{1}\left[t_{1}^{1}, t_{1}^{2}\right] \cup\left\{(p, q),\left(s_{1}, t_{1}^{1}\right),\left(s_{1}, t_{1}^{2}\right)\right\}$ if $p=t_{1}^{2}$, and let $\Sigma$ be $O_{1}\left[t_{1}^{3}, p\right] \cup\left\{(p, q),\left(s_{1}, t_{1}^{1}\right),\left(s_{1}, t_{1}^{3}\right)\right\}$ if $p \neq t_{1}^{2}$ and $q \in O_{1}\left[t_{1}^{1}, p\right)$. Then $\Sigma$ is a gear in $G$ with fewer vertices than $H$, a contradiction.

So symmetry allows us to assume that one of $p$ and $q$ is on $O_{1}\left(t_{1}^{1}, t_{1}^{2}\right)$ and the other is on $O_{1}\left(t_{1}^{2}, t_{1}^{3}\right)$. Let $\Sigma$ be obtained from $H \cup\left\{\left(s_{1}, t_{1}^{3}\right)\right\}$ by deleting all vertices on $O_{1}(p, q)$ if $p \in O_{1}\left(t_{1}^{1}, t_{1}^{2}\right)$, and let $\Sigma$ be $O_{1}\left[t_{1}^{1}, p\right] \cup\left\{(p, q),\left(s_{1}, t_{1}^{1}\right),\left(s_{1}, t_{1}^{2}\right)\right\}$ otherwise. Then $\Sigma$ is a gear in $G$ with fewer vertices than $H$, again a contradiction.

Case 2. $H$ has at least two distinguished vertices.

Recall the construction of $H$ and the three operations performed with respect to each sink $t_{i}$, set $P_{i}=\emptyset$ if the first and the third operations are applied, and set $t_{i}^{1}=t_{i}^{2}=t_{i}$ if the first and second operations are applied. Renaming the subscripts if necessary, we assume that $s_{1}, t_{1}^{1}, t_{1}^{2}, s_{2}, t_{2}^{1}, t_{2}^{2}, \ldots, s_{k}, t_{k}^{1}, t_{k}^{2}$ occur on $C$ in order if we traverse $C$ from $s_{1}$ in the clockwise direction. Moreover, let $Q_{i}=t_{i}$ if the first and second operations are applied and let $Q_{i}$ be the segment of $O_{i}$ from $t_{i}^{1}$ to $t_{i}^{2}$ if the third operations are applied. Then the $\operatorname{arcs}$ on $C$ and on all these $Q_{i}$ form a circuit in $H$, denoted by $D$. Given two vertices $p$ and $q$ on $D$, we use $D[p, q]$ to denote the segment of $D$ from $p$ to $q$ in the clockwise direction.

Let us now construct a subgraph $\Sigma$ of $H \cup\left\{\left(s_{1}, v\right)\right\}$ as follows.

When $v \in D\left[s_{i}, t_{i}^{1}\right]$ for some $i$ with $1 \leq i \leq k$, let $H^{\prime}$ be the digraph obtained from $H \cup\left\{\left(s_{1}, v\right)\right\}$ by deleting all vertices on $D\left(s_{1}, v\right)$, and let $\Sigma$ be the component of $H^{\prime}$ containing $s_{1}$.

When $v \in D\left[t_{i}^{2}, s_{i+1}\right)$ for some $i$ with $1 \leq i \leq k$, where $s_{k+1}=s_{1}$, let $H^{\prime}$ be the digraph obtained from $H \cup\left\{\left(s_{1}, v\right)\right\}$ by deleting all vertices on $D\left(v, s_{1}\right)$, and let $\Sigma$ be the component of $H^{\prime}$ containing $s_{1}$.

When $v \in P_{i} \cup O_{i} \backslash\left\{t_{i}^{1}, t_{i}^{2}\right\}$ for some $i$ with $1 \leq i \leq k$, let $R$ stand for one of the segments $D\left(s_{1}, t_{i}^{1}\right)$ and $D\left(t_{i}^{2}, s_{1}\right)$ that contains a distinguished vertex $s_{j}$ with $2 \leq j \leq k$, let $H^{\prime}$ be the digraph obtained from $H \cup\left\{\left(s_{1}, v\right)\right\}$ by deleting all vertices on $R$, and let $\Sigma$ be the component of $H^{\prime}$ containing $s_{1}$.

It is easy to see that $\Sigma$ is a gear in $G$ with fewer vertices than $H$ in every subcase; this contradiction completes the proof of the present lemma.

\section{Structures}

Let $Q, P_{1}, P_{2}$ be three directed paths such that the ends $s_{i}, t_{i}$ of $P_{i}$ are both on $Q$ for $i=1,2$ and that $P_{1}\left(s_{1}, t_{1}\right), P_{2}\left(s_{2}, t_{2}\right)$, and $Q$ are pairwise vertex-disjoint. We say that $P_{1}$ and $P_{2}$ cross with respect to $Q$ if $s_{1}, t_{1}, s_{2}, t_{2}$ are distinct and each segment of $Q$ (possibly $Q$ is a cycle) between $s_{1}$ and $t_{1}$ contains precisely one of $s_{2}$ and $t_{2}$.

For convenience, we call a digraph $G$ permissible hereafter if $G$ contains no subgraph isomorphic to an odd cycle, a gear, or a ring. With this notion, Theorem 1.5 amounts to saying that a digraph is kernel ideal (also kernel Mengerian) if and only if it is permissible. The following theorem aims to give a construction of strongly connected components of permissible digraphs, and exhibit some important properties enjoyed by them, where a cycle $C$ in $G$ is called induced 
if no arc outside $C$ in $G$ connects two vertices on $C$. Roughly speaking, every strongly connected permissible digraph admits an ear decomposition (see, for instance, [3]) such that, except the initial cycle ear, each ear (cycle or path) is a child of a unique parent ear. Furthermore, each such ear is subject to strict restrictions on the locations of its ends.

Theorem 4.1. Let $G=(V, A)$ be a strongly connected permissible digraph, where $|V| \geq 2$. Then $G$ has a rooted tree-like structure (see Figure 3 for an illustration); that is, there exists a rooted tree $T$, such that

(i) the root $r$ of $T$ corresponds to an (arbitrarily given) induced cycle $P_{r}$ of $G$;

(ii) each $v \in V(T) \backslash r$ corresponds to an $s_{v}$ - $t_{v}$ path $P_{v}$ (possibly $s_{v}=t_{v}$ ), such that if $u$ is the parent of $v$ in $T$, then both $s_{v}$ and $t_{v}$ are on $P_{u}$, and at least one of $s_{v}$ and $t_{v}$ is on $P_{u}\left(s_{u}, t_{u}\right)$ if $u \neq r$;

(iii) $P_{r}$ and $P_{v}\left(s_{v}, t_{v}\right)$ for all $v \in V(T) \backslash r$ are pairwise vertex-disjoint, and $G$ is the union of $P_{v}$ for all $v \in V(T)$;

(iv) for any $v \in V(T) \backslash r$ and any child $x$ of $v$, if $t_{x} \in P_{v}\left(s_{x}, t_{v}\right]$, then $s_{x}=s_{v}$;

(v) for any $v \in V(T) \backslash r$ and any child $x$ of $v$, if $t_{x} \in P_{v}\left[s_{v}, s_{x}\right)$, then $t_{x} \neq s_{v}$. Also, $s_{x} \neq t_{v}$ if $s_{v} \neq t_{v}$

(vi) for any $v \in V(T)$ and any two children $x, y$ of $v$, paths $P_{x}$ and $P_{y}$ do not cross with respect to $P_{v}$;

(vii) for any $v \in V(T) \backslash r$ and any two children $x, y$ of $v$, if $s_{x}=s_{v}$, then $s_{y} \in P_{v}\left[s_{v}, t_{x}\right)$;

(viii) for any $v \in V(T) \backslash r$ and any two children $x, y$ of $v$, if $t_{x} \in P_{v}\left[s_{v}, s_{x}\right)$ and $t_{y} \in P_{v}\left[s_{v}, s_{y}\right]$, then $s_{y} \notin P_{v}\left[t_{x}, s_{x}\right)$;

(ix) for any two children $x, y$ of $r$, if $s_{x} \neq t_{x}$ and $s_{x}, t_{x}, t_{y}, s_{y}$ occur on $P_{r}$ in order when we traverse $P_{r}$ in its direction from $s_{x}$, then $s_{y}=s_{x}$; and

(x) for any two children $x, y$ of $r$, if $s_{x} \neq t_{x}$ and $s_{x}, t_{y}, s_{y}, t_{x}$ occur on $P_{r}$ in order when we traverse $P_{r}$ in its direction from $s_{x}$, then precisely one of the following three cases occurs:

- both $s_{y}$ and $t_{y}$ are on $P_{r}\left(s_{x}, t_{x}\right)$;

- $s_{x}=s_{y}=t_{y}$;

- $s_{y}=t_{x}, t_{y}=s_{x}$, and $\left|P_{r}\left[s_{x}, s_{y}\right]\right|$ is even.

The following two lemmas will be used repeatedly in the proof of this theorem.

Lemma 4.2. Let $H$ be obtained from a directed cycle $C$ by adding an $s_{1}-t_{1}$ path $P_{1}$ (possibly $\left.s_{1}=t_{1}\right)$ and an $s_{2}-t_{2}$ path $P_{2}$, such that

- $P_{1}\left(s_{1}, t_{1}\right), P_{2}\left(s_{2}, t_{2}\right)$, and $C$ are pairwise vertex-disjoint;

- $P_{1} \cup P_{2} \cup C$ is even and contains no parallel arcs; and

- $s_{1}$ and $t_{1}$ are on $C$, and one of $s_{2}$ and $t_{2}$ is on $P_{1}\left(s_{1}, t_{1}\right)$, the other is in $C \backslash\left\{s_{1}, t_{1}\right\}$.

Then $H$ contains a gear.

Proof. Symmetry allows us to assume that $s_{2}$ is on $P_{1}\left(s_{1}, t_{1}\right)$ if $s_{1}=t_{1}$. Let $F$ be the digraph obtained from $H$ by first deleting all arcs on $P_{1}\left[s_{1}, s_{2}\right]$ if $s_{2}$ is on $P_{1}\left(s_{1}, t_{1}\right)$, by first deleting all arcs on $C\left[s_{1}, s_{2}\right]$ if $s_{2}$ is on $C\left(s_{1}, t_{1}\right)$, and by first deleting all $\operatorname{arcs}$ on $C\left[s_{2}, s_{1}\right]$ if $s_{2}$ is on $C\left(t_{1}, s_{1}\right)$, and then deleting all resulting isolated vertices (if any). Then $F$ is a gear in each case. 


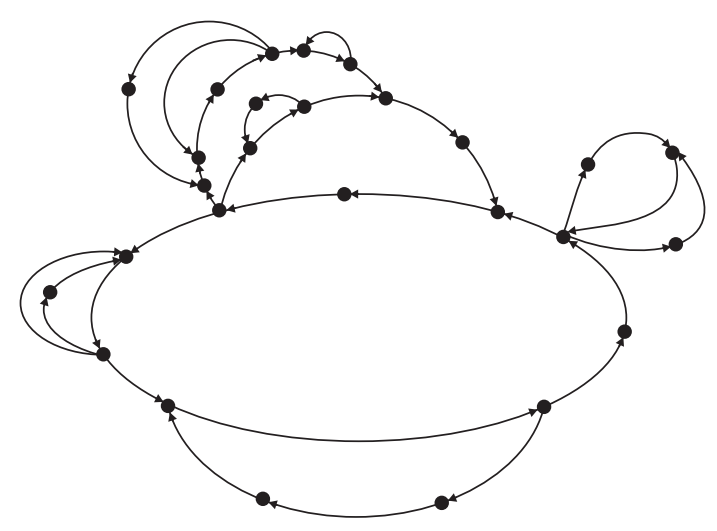

Figure 3: A rooted tree-like structure.

Lemma 4.3. Let $H$ be obtained from a directed cycle $C$ by adding an $s_{i}-t_{i}$ path $P_{i}$ for $i=1,2$, where $s_{i}$ and $t_{i}$ are two distinct vertices on $C$, such that

- $P_{1}\left(s_{1}, t_{1}\right), P_{2}\left(s_{2}, t_{2}\right)$, and $C$ are pairwise vertex-disjoint;

- $P_{1} \cup P_{2} \cup C$ is even and contains no parallel arcs; and

- $P_{1}$ and $P_{2}$ cross with respect to $C$.

Then $H$ contains a gear.

Proof. Swapping the subscripts if necessary, we may assume that $s_{1}, s_{2}, t_{1}, t_{2}$ occur on $C$ in order if we traverse $C$ in its direction. Thus the digraph obtained from $H$ by first deleting all arcs on $C\left[s_{1}, s_{2}\right]$ and then deleting all resulting isolated vertices (if any) is a gear.

Proof of Theorem 4.1. We shall first construct a rooted tree $T$ and paths $P_{v}$ for all $v \in V(T)$ with properties (i)-(iii), and then demonstrate that they enjoy properties (iv)-(x) as well.

In the initialization step, we take an arbitrary induced cycle $P_{r}$ in $G$ (which is even), and construct a rooted tree $T$ consisting of the root $r$ only, which corresponds to $P_{r}$. At a general step, suppose we have constructed a rooted tree $T$ and paths $P_{v}$ for all $v \in V(T)$ with the properties as specified in (i), (ii), and (iii). If $G$ is already the union of $P_{v}$ for all $v \in V(T)$, then we are done. Otherwise, we augment $T$ by adding a new vertex $v$ and construct $P_{v}$ with the desired properties.

Let us make some observations about the current $T$ and $P_{v}$ for $v \in V(T)$ before describing the construction procedure. Throughout we use $R_{v}$ to denote the path from $r$ to each vertex $v$ in $T$.

(1) For each $v \in V(T)$, the union $\cup_{z \in V\left(R_{v}\right)} P_{z}$ is strongly connected and hence has a cycle containing $P_{v}$.

To justify this, we apply induction on $\left|R_{v}\right|$. If $\left|R_{v}\right|=0$; that is, $v=r$, then (1) is trivial because $P_{r}$ is a cycle. So we proceed to the induction step. Let $u$ be the parent of $v$ in $T$. By induction hypothesis, $\cup_{z \in V\left(R_{u}\right)} P_{z}$ is strongly connected. Since $P_{v}$ is a path with ends in $\cup_{z \in V\left(R_{u}\right)} P_{z}$, it is a routine matter to check that $\cup_{z \in V\left(R_{v}\right)} P_{z}$ is also strongly connected. Thus (1) holds. 
(2) For each $v \in V(T)$, if $G$ has a path $Q$ whose one end $t$ is on $P_{v}\left(s_{v}, t_{v}\right)$, the other end $s$ is on $P_{u}$ but outside $P_{v}$ for some ancestor $u$ of $v$ in $T$, and vertices in $V(Q) \backslash\{s, t\}$ are all outside $\cup_{z \in V\left(R_{v}\right)} P_{z}$, then $G$ contains a gear.

We prove (2) by induction on $|V(T)|$. Since the statement follows instantly from Lemma 4.2 when $|V(T)|=2$, we proceed to the induction step. Let $a$ be the parent of $v$. We may assume that

(3) $s \notin P_{a}$ and $s_{v} \neq t_{v}$.

Otherwise, $s \in P_{a}$ or $s_{v}=t_{v}$. By (1), $P_{a}$ is contained in a cycle $C$ in $\cup_{z \in V\left(R_{a}\right)} P_{z}$. If $s \in P_{a}$, then $s \in C$; set $L=\emptyset$ in this case. If $s_{v}=t_{v}$ while $s \notin P_{a}$ then, since $\cup_{z \in V\left(R_{a}\right)} P_{z}$ is strongly connected and since $s_{v}$ is on $P_{a}\left(s_{a}, t_{a}\right)$ (recall property (ii)), there exists a directed path $L$ from $C$ to $s$ in $\cup_{z \in V\left(R_{a}\right)} P_{z}$, such that $L \cup Q$ is a directed path in $G$ which has only two ends in $C \cup P_{v}$ and has one end in $C \backslash s_{v}$. Applying Lemma 4.2 with respect to $P_{v}, L \cup Q$, and $C$, we deduce that $G$ contains a gear, so (3) holds.

(4) $s_{v}$ is on $P_{a}\left[t_{v}, t_{a}\right]$.

Otherwise, $s_{v}$ is on $P_{a}\left[s_{a}, t_{v}\right)$. Let $P_{v^{\prime}}$ denote the path obtained from $P_{a}$ by replacing $P_{a}\left[s_{v}, t_{v}\right]$ with $P_{v}$, let $R_{v^{\prime}}$ be the path obtained from $R_{v}$ by contracting the edge $v a$ into a single vertex $v^{\prime}$, and let $T^{\prime}$ be the rooted tree consisting of $R_{v^{\prime}}$ only. By (3), we have $s \notin P_{v^{\prime}}$. From the induction hypothesis on $T^{\prime}$, it follows that $G$ contains a gear, so (4) is justified.

(5) $t$ is the origin of $Q$.

Otherwise, $t$ is the terminus of $Q$. By (4), $P_{v} \cup P_{a}$ has a cycle $C$ containing $P_{v}$. Since $\cup_{z \in V\left(R_{a}\right)} P_{z}$ is strongly connected, it contains a directed path $L$ from $s$ to $C$. Thus $Q, L$, and $C$ form a gear in $G$. This proves (5).

Let $Q^{\prime}$ stand for the path $P_{v}\left[s_{v}, t\right] \cup Q$. Then $Q^{\prime}$ is a directed path from $s_{v}$ to $s$ whose internal vertices are all outside $\cup_{z \in V\left(R_{a}\right)} P_{z}$. If $s_{v} \notin\left\{s_{a}, t_{a}\right\}$, then (3) and the induction hypothesis with respect to the path $Q^{\prime}$ and rooted tree $T^{\prime}=R_{a}$ imply that $G$ contains a gear. So we may assume that $s_{v} \in\left\{s_{a}, t_{a}\right\}$. In view of (3) and (4), we have $s_{v}=t_{a}$. Hence $t_{v}$ is on $P_{a}\left(s_{a}, t_{a}\right)$ by property (ii). Since $\cup_{z \in V\left(R_{a}\right)} P_{z}$ is strongly connected, it has a cycle $C$ containing $P_{a}$ and a directed path $L$ from $s$ to $C$, such that $Q \cup L$ is a directed path in $G$ which has only one end in $C$. Thus $P_{v}\left[t, t_{v}\right], Q \cup L$, and $C$ form a gear in $G$, completing the proof of (2).

(6) Suppose $G$ has a path $Q$ whose ends $s$ and $t$ (possibly $s=t$ ) are the only vertices of $Q$ in $\cup_{z \in V(T)} P_{z}$. Let $v$ be a vertex in $T$ such that at least one of $s$ and $t$ is on $P_{v}\left(s_{v}, t_{v}\right)$ and that no descendant of $v$ in $T$ has this property, where $P_{v}\left(s_{v}, t_{v}\right)=P_{r}$ if $v=r$. Then both $s$ and $t$ are on $P_{v}$.

To justify this, assume the contrary: $t$ is on $P_{v}\left(s_{v}, t_{v}\right)$ while $s$ is outside $P_{v}$ (renaming the ends of $Q$ if necessary). By (2), we obtain

(7) $s$ is not on $P_{z}$ for any ancestor $z$ of $v$ in $T$.

Observe that

(8) $s$ is not on $P_{z}$ for any descendant $z$ of $v$ in $T$ either.

Otherwise, from the hypothesis on $v$, we deduce that $s \in\left\{s_{z}, t_{z}\right\}$. So $s$ is on $P_{a}$, where $a$ is the parent of $z$ in $T$. Once again by the hypothesis on $v$, we have $s \in\left\{s_{a}, t_{a}\right\}$ if $a \neq v$. Let us proceed in this way and eventually we see that $s$ is on $P_{v}$, this contradiction justifies (8).

From (7), (8), and property (ii), we deduce that $T$ has a vertex $u$ such that $s$ is on $P_{u}\left(s_{u}, t_{u}\right)$ and that neither vertex in $\{u, v\}$ is a descendant of the other. Symmetry allows us to assume 
that $t$ is the terminus of $Q$. Let $C$ be a directed cycle containing $P_{v}$ in $\cup_{z \in V\left(R_{v}\right)} P_{z}$ and let $a$ be the parent of $v$ in $T$. Since $\cup_{z \in V\left(R_{a} \cup R_{u}\right)} P_{z}$ is also strongly connected, it contains a directed path $L$ from $s$ to $C$. Thus $Q, L$, and $C$ form a gear in $G$. Therefore (6) is established.

Now we are ready to describe the construction procedure. Suppose $G$ is not the union of $P_{v}$ for all vertices $v$ in the current $T$. Then the strong connectedness of $G$ guarantees the existence of a path $Q$ as specified in (6). Let $v$ be as defined in (6). We augment $T$ by adding a vertex $z$ and an edge $v z$ and set $P_{z}=Q$. The process is repeated until $G$ is the union of $P_{v}$ for all $v \in V(T)$.

It remains to show that the rooted tree $T$ and paths $P_{v}$ enjoy all the desired properties. In our proofs of (iv)-(viii), we reserve the symbol $u$ for the parent of the vertex $v$ specified in the statements, and the symbol $C_{a}$ for a cycle containing $P_{a}$ in $\cup_{z \in V\left(R_{a}\right)} P_{z}$ (see (1)) for each vertex $a$ on $T$.

(iv) If $s_{x} \neq s_{v}$, then $P_{x} \cup P_{v}\left[s_{x}, t_{v}\right] \cup C_{u}$ would be a gear; this contradiction justifies (iv).

(v) Recall (ii), if one of $s_{x}$ and $t_{x}$ is in $\left\{s_{v}, t_{v}\right\}$, then the other is on $P_{v}\left(s_{v}, t_{v}\right)$. Set $H=$ $P_{x} \cup P_{v}\left[s_{x}, t_{v}\right] \cup C_{u}$ if $t_{x}=s_{v}$, and set $H=P_{x} \cup P_{v} \cup C_{u}\left[s_{v}, t_{v}\right]$ if $s_{x}=t_{v} \neq s_{v}$. Then $H$ is a gear in either case and hence (v) holds.

(vi) Applying Lemma 4.3 to $C_{v} \cup P_{x} \cup P_{y}$, we deduce instantly that $P_{x}$ and $P_{y}$ do not cross with respect to $P_{v}$.

(vii) Assume the contrary: $s_{y} \notin P_{v}\left[s_{v}, t_{x}\right)$. By (iv), $t_{y} \notin P_{v}\left(s_{y}, t_{v}\right]$. So $t_{y} \in P_{v}\left(s_{v}, s_{y}\right]$ (see (v)). Set $H=P_{v}\left[s_{v}, t_{x}\right] \cup P_{x} \cup P_{y}$ if $t_{y} \in P_{v}\left(s_{v}, t_{x}\right)$, and set $H=P_{v}\left[s_{v}, s_{y}\right] \cup P_{x} \cup P_{y}$ if $t_{y} \in P_{v}\left[t_{x}, s_{y}\right]$. Notice that, by (vi), if $t_{y} \in P_{v}\left(s_{v}, t_{x}\right)$, then $s_{y}=t_{x}$, and that if $s_{v}=t_{v}$, then $s_{y} \neq t_{v}$ by the assumption. Consequently, $H$ is a gear in either case, a contradiction. So (vii) is justified.

(viii) Assume the contrary: $s_{y} \in P_{v}\left[t_{x}, s_{x}\right)$. Note that if $s_{y} \neq t_{x}$, then $t_{y} \in P_{v}\left[t_{x}, s_{y}\right]$ by (vi). Set $H=P_{x} \cup C_{v}\left[s_{x}, s_{y}\right] \cup P_{y}$ if $s_{y} \neq t_{x}$, and set $H=P_{x} \cup C_{v}\left[s_{x}, t_{x}\right] \cup P_{y}$ if $s_{y}=t_{x}$. By (v), we have $t_{x} \neq s_{v}$. Besides, (v) implies $t_{y} \neq s_{v}$ when $t_{x}=s_{y} \neq t_{y}$. It follows that $H$ is a gear in either case; this contradiction proves (viii).

(ix) If $s_{y} \neq s_{x}$, then $P_{r}\left[s_{x}, s_{y}\right] \cup P_{x} \cup P_{y}$ would be a gear, a contradiction.

(x) If $t_{x}=s_{y}$ but $s_{x} \neq t_{y}$, then $H=P_{x} \cup P_{y} \cup P_{r}\left[s_{x}, s_{y}\right]$ would be a gear. If $s_{x}=t_{y}, t_{x}=s_{y}$, but $P_{r}\left[s_{x}, s_{y}\right]$ is odd, then $P_{r} \cup P_{x} \cup P_{y}$ would be a ring. Using symmetry, it is easy to see that precisely one of the three stated cases can occur, completing the proof.

Given a digraph $G$, we use $G^{s}$ to denote the digraph obtained from $G$ by contracting each strongly connected component of $G$ into a single vertex, such that the number of arcs between any two vertices in $G^{s}$ equals the number of arcs between the corresponding strongly connected components of $G$. We call $G^{s}$ the skeleton of $G$. Clearly, $G^{s}$ contains no directed cycles but it may contain parallel arcs. Recall that a digraph is called weakly connected if its underlying graph is connected.

Theorem 4.4. Let $G$ be a weakly connected digraph with no gear. Suppose each sink of $G^{s}$ corresponds to a strongly connected component of $G$ with at least two vertices. Then the underlying graph of $G^{s}$ is a tree. In particular, there is at most one arc between any two strongly connected components of $G$. 
Proof. Since $G^{s}$ is acyclic, we have

(1) $G^{s}$ contains a path from each vertex $u$ to some $\operatorname{sink}$ of $G^{s}$.

Suppose on the contrary that the underlying graph of $G^{s}$ is not a tree. Then $G^{s}$ contains at least one circuit; let $C$ be such a circuit with the smallest number of sinks (possibly $C$ is formed by two parallel arcs). Notice that

(2) there is no path $P$ from a sink of $C$ to some other vertex on $C$ in $G^{s}$, such that the internal vertices of $P$ are all outside $C$.

Otherwise, it is a routine matter to check that $C \cup P$ contains either a directed cycle or a circuit with fewer sinks than $C$, this contradiction justifies (2).

Let $t_{1}, t_{2}, \ldots, t_{k}$ be all the sinks of $C$. By (1), $G^{s}$ contains a path $P_{i}$ from $t_{i}$ to some sink $u_{i}$ of $G^{s}$, for each $i=1,2, \ldots, k$.

(3) $C$ and $P_{i} \backslash t_{i}, i=1,2, \ldots, k$, are pairwise vertex-disjoint.

Indeed, from (2) we see that $P_{i} \backslash t_{i}$ is vertex-disjoint from $C$ for $1 \leq i \leq k$. Also, $P_{i}$ and $P_{j}$ are vertex-disjoint whenever $i \neq j$, for otherwise let $x$ be a common vertex of $P_{i}$ and $P_{j}$. Then $C \cup P_{i}\left[t_{i}, x\right] \cup P_{j}\left[t_{j}, x\right]$ would contain a circuit with fewer sinks than $C$, a contradiction. So (3) holds.

We propose to show that a gear in $G$ can be obtained from $C \cup\left(\cup_{i=1}^{k} P_{i}\right)$ by blowing up its vertices.

To this end, let $\Omega_{u}$ denote the strongly connected component of $G$ corresponding to each vertex $u$ in $G^{s}$. For each vertex $v$ of $C$ (resp. each degree-two vertex $v$ in $\cup_{i=1}^{k} P_{i}$ ), let $e_{v}^{1}$ and $e_{v}^{2}$ denote the two arcs of $C$ (resp. of $\cup_{i=1}^{k} P_{i}$ ) incident with $v$, where $e_{v}^{1}$ enters $v$ if $v$ is not a source of $C$. We also view $e_{v}^{1}$ and $e_{v}^{2}$ as two $\operatorname{arcs}$ in $G$; in this situation, we use $v^{j}$ to denote the end of $e_{v}^{j}$ in $\Omega_{v}$ for $j=1,2$.

For each vertex $v$ on $C$ that is not a sink of $C$ and for each degree-two vertex $v$ on $\cup_{i=1}^{k} P_{i}$, let $Q_{v}$ be a path from $v^{1}$ to $v^{2}$ in $\Omega_{v}$. Let $Q$ be the union of $Q_{v}$ 's for all vertices $v$ in $C \cup\left(\cup_{i=1}^{k} P_{i}\right)$.

For each sink $t_{i}$ on $C$ with $t_{i}=u_{i}$ (that is, $P_{i}$ consists of a single vertex), let $R_{i}^{j}$ be a path in $\Omega_{t_{i}}$ ) from $t_{i}^{j}$ to $t_{i}^{2-j}$ for $j=1,2$, such that at least one of $R_{i}^{1}$ and $R_{i}^{2}$ has positive length (such paths exist because $\Omega_{t_{i}}$ contains at least two vertices by hypothesis). Let $O_{i}$ be the directed cycle in $R_{i}^{1} \cup R_{i}^{2}$ containing $t_{i}^{1}$ and set $Q_{i}=O_{i} \cup R_{i}^{2}$.

For each sink $t_{i}$ on $C$ with $t_{i} \neq u_{i}$ (that is, $P_{i}$ contains at least two vertices), we assume for simplicity that $t_{i}$ (resp. $u_{i}$ ) is the end of the starting (resp. ending) arc of $P_{i}$ contained in $\Omega_{t_{i}}$ (resp. $\Omega_{u_{i}}$ ). Let $R_{i}^{1}$ be a path in $\Omega_{t_{i}}$ from $t_{i}^{1}$ to $t_{i}$, and let $R_{i}^{2}$ be a path in $\Omega_{t_{i}}$ from $t_{i}^{2}$ to $R_{i}^{1}$. Let $O_{i}$ be a cycle passing through $u_{i}$ in $\Omega_{u_{i}}$ and set $Q_{i}=O_{i} \cup R_{i}^{1} \cup R_{i}^{2}$.

From (3) it follows instantly that the arcs of $G$ in $C \cup Q \cup\left(\cup_{i=1}^{k} P_{i} \cup Q_{i}\right)$ form a gear in $G$, this contradiction implies that the underlying graph of $G^{s}$ must be a tree.

\section{Reductions}

In this section we introduce a series of reduction operations, under which the integrality properties enjoyed by the fractional kernel polytope and its defining system are preserved. Our reductions are all based on the assumption that every permissible digraph with fewer vertices or with fewer arcs than the input digraph $G$ is kernel ideal and kernel Mengerian (see Theorem 1.5). 
For any real number $\alpha$, we shall use $[\alpha]^{+}$as a shorthand for $\max \{0, \alpha\}$. We shall also use $\phi_{v}(\boldsymbol{y}, \boldsymbol{z})$ to denote the left-hand side of (1.8) throughout.

\section{Reduction 1}

Instance: A digraph $G=(V, A)$ with a sink $a$ and a weight function $\boldsymbol{w} \in \mathbb{Z}^{V}$.

Description: Let $G^{\prime}=\left(V^{\prime}, A^{\prime}\right)$ be the digraph obtained from $G$ by deleting all vertices in $\{a\} \cup N_{G}^{-}(a)$ and let $\boldsymbol{w}^{\prime}$ be the restriction of $\boldsymbol{w}$ to $\mathbb{Z}^{V^{\prime}}$. Suppose $\boldsymbol{x}^{\prime}$ and $\left(\boldsymbol{y}^{\prime}, \boldsymbol{z}^{\prime}\right)$ are integral optimal solutions to $\mathbb{P}\left(G^{\prime}, \boldsymbol{w}^{\prime}\right)$ and $\mathbb{D}\left(G^{\prime}, \boldsymbol{w}^{\prime}\right)$, respectively. Let $e_{i}=\left(b_{i}, a\right)$ for $1 \leq i \leq k$ be all the arcs incident with $a$, and set $\alpha_{i}=z^{\prime}\left(N_{G}^{-}\left(b_{i}\right) \cap V^{\prime}\right)$ for $1 \leq i \leq k$, where $z^{\prime}(\emptyset)=0$. Define

- $x^{*}(a)=1$ and $x^{*}\left(b_{i}\right)=0$ for $1 \leq i \leq k$;

- $y^{*}\left(e_{1}\right)=[-w(a)]^{+}+\left[-w\left(b_{1}\right)\right]^{+}+\alpha_{1}, y^{*}\left(e_{i}\right)=\left[-w\left(b_{i}\right)\right]^{+}+\alpha_{i}$ for $2 \leq i \leq k$, and $y^{*}(e)=0$ for all $e \notin A^{\prime} \cup\left\{e_{1}, e_{2}, \ldots, e_{k}\right\}$;

- $z^{*}(a)=w(a)+\sum_{i=1}^{k} y^{*}\left(e_{i}\right)$ and $z^{*}\left(b_{i}\right)=0$ for $1 \leq i \leq k$; and

- $x^{*}(v)=x^{\prime}(v)$ for all $v \in V^{\prime}, y^{*}(e)=y^{\prime}(e)$ for all $e \in A^{\prime}$, and $z^{*}(v)=z^{\prime}(v)$ for all $v \in V^{\prime}$.

Lemma 5.1. For Reduction 1, the following statements hold:

(i) $\boldsymbol{x}^{*}$ and $\left(\boldsymbol{y}^{*}, \boldsymbol{z}^{*}\right)$ are integral optimal solutions to $\mathbb{P}(G, \boldsymbol{w})$ and $\mathbb{D}(G, \boldsymbol{w})$, respectively; and

(ii) the optimal value of $\mathbb{P}(G, \boldsymbol{w})$ equals $w\left(\right.$ a) plus that of $\mathbb{P}\left(G^{\prime}, \boldsymbol{w}^{\prime}\right)$.

Proof. Clearly, $\boldsymbol{x}^{*}$ satisfies $(1.2)-(1.4)$. Since $w(a)+[-w(a)]^{+}=[w(a)]^{+} \geq 0$, we have $z^{*}(a) \geq 0$. It is then easy to see that $\left(\boldsymbol{y}^{*}, \boldsymbol{z}^{*}\right)$ satisfies (1.8) - (1.10). So $\boldsymbol{x}^{*}$ and $\left(\boldsymbol{y}^{*}, \boldsymbol{z}^{*}\right)$ are integral feasible solutions to $\mathbb{P}(G, \boldsymbol{w})$ and $\mathbb{D}(G, \boldsymbol{w})$, respectively. By the above definition, we have $\boldsymbol{w}^{T} \boldsymbol{x}^{*}=w(a)+\left(\boldsymbol{w}^{\prime}\right)^{T} \boldsymbol{x}^{\prime}=w(a)-y^{\prime}\left(A^{\prime}\right)+z^{\prime}\left(V^{\prime}\right)=z^{*}(a)-\sum_{i=1}^{k} y^{*}\left(e_{i}\right)-y^{\prime}\left(A^{\prime}\right)+z^{\prime}\left(V^{\prime}\right)=$ $-y^{*}(A)+z^{*}(V)$. From the LP-duality theorem, we thus deduce that $\boldsymbol{x}^{*}$ and $\left(\boldsymbol{y}^{*}, \boldsymbol{z}^{*}\right)$ are optimal solutions to $\mathbb{P}(G, \boldsymbol{w})$ and $\mathbb{D}(G, \boldsymbol{w})$, respectively. Hence both (i) and (ii) are established.

\section{Reduction 2}

Instance: A permissible digraph $G=(V, A)$ with a weight function $\boldsymbol{w} \in \mathbb{Z}^{V}$, and three distinct vertices $a, b, c$ of $G$, such that $a$ and $c$ are nonadjacent and have no common in-neighbor, $N_{G}^{+}(a)=$ $\{b\}$, and $N_{G}^{+}(b)=\{c\}(\operatorname{arc}(c, b)$ may exist).

Description: Let $G^{\prime}=\left(V^{\prime}, A^{\prime}\right)$ be the digraph obtained from $G$ by first deleting the arc $(a, b)$ and then identifying $a$ with $c$ (we still use $c$ to denote the resulting vertex), and let $w^{\prime}(c)=w(a)+w(c)$ and $w^{\prime}(v)=w(v)$ if $v \in V^{\prime} \backslash c$. Suppose $\boldsymbol{x}^{\prime}$ and $\left(\boldsymbol{y}^{\prime}, \boldsymbol{z}^{\prime}\right)$ are integral optimal solutions to $\mathbb{P}\left(G^{\prime}, \boldsymbol{w}^{\prime}\right)$ and $\mathbb{D}\left(G^{\prime}, \boldsymbol{w}^{\prime}\right)$, respectively. Set $\alpha=\sum_{v \in N_{G}^{-}(a)} y^{\prime}((v, a)), \beta=z^{\prime}\left(N_{G}^{-}(a)\right)$, and $\gamma=w(a)+\alpha-\beta$.

- $x^{*}(a)=x^{\prime}(c)$, and $x^{*}(v)=x^{\prime}(v)$ for every $v \in V \backslash a$;

- $y^{*}((a, b))=[-\gamma]^{+}, y^{*}((b, c))=y^{\prime}((b, c))+[\gamma]^{+}$, and $y^{*}(e)=y^{\prime}(e)$ for every $e \in A \backslash\{(a, b),(b, c)\}$; and

- $z^{*}(a)=[\gamma]^{+}, z^{*}(b)=z^{\prime}(b)+[-\gamma]^{+}$, and $z^{*}(v)=z^{\prime}(v)$ for every $v \in V \backslash\{a, b\}$.

Lemma 5.2. For Reduction 2, the following statements hold:

(i) $G^{\prime}$ is also permissible; 
(ii) $\boldsymbol{x}^{*}$ and $\left(\boldsymbol{y}^{*}, \boldsymbol{z}^{*}\right)$ are integral optimal solutions to $\mathbb{P}(G, \boldsymbol{w})$ and $\mathbb{D}(G, \boldsymbol{w})$, respectively; and (iii) the optimal value of $\mathbb{P}(G, \boldsymbol{w})$ equals that of $\mathbb{P}\left(G^{\prime}, \boldsymbol{w}^{\prime}\right)$.

Proof. Suppose on the contrary that $G^{\prime}$ contains a subgraph $\Sigma$ which is isomorphic to an odd cycle, or a gear, or a ring. It is then a routine matter to check that the subgraph of $G$ induced by all arcs in $\Sigma \cup\{(a, b),(b, c)\}$ would contain an odd cycle, or a gear, or a ring, contradicting the hypothesis that $G$ is permissible. So (i) holds.

To prove the remaining two statements, observe that

- $x^{*}(a)+x^{*}(b)=x^{*}(b)+x^{*}(c)=x^{\prime}(b)+x^{\prime}(c) \leq 1$

- $\phi_{a}\left(\boldsymbol{y}^{*}, \boldsymbol{z}^{*}\right)=-\sum_{a \in e} y^{*}(e)+z^{*}(a)+z^{*}\left(N_{G}^{-}(a)\right)=-\alpha-y^{*}((a, b))+[\gamma]^{+}+\beta=-\alpha-[-\gamma]^{+}+$ $[\gamma]^{+}+\beta=-\alpha+\gamma+\beta=w(a)$

- $\phi_{b}\left(\boldsymbol{y}^{*}, \boldsymbol{z}^{*}\right)=-\sum_{b \in e} y^{*}(e)+z^{*}(b)+z^{*}\left(N_{G}^{-}(b)\right)=\left(-y^{*}((a, b))-\sum_{b \in e} y^{\prime}(e)-[\gamma]^{+}\right)+\left(z^{\prime}(b)+\right.$ $\left.[-\gamma]^{+}\right)+z^{*}(a)+z^{\prime}\left(N_{G^{\prime}}^{-}(b)\right)=-\sum_{b \in e} y^{\prime}(e)+z^{\prime}(b)+z^{\prime}\left(N_{G^{\prime}}^{-}(b)\right) \leq w^{\prime}(b)=w(b)$; and

- $\phi_{c}\left(\boldsymbol{y}^{*}, \boldsymbol{z}^{*}\right)=-\sum_{c \in e} y^{*}(e)+z^{*}(c)+z^{*}\left(N_{G}^{-}(c)\right) \leq\left(-\sum_{c \in e} y^{\prime}(e)+\alpha-[\gamma]^{+}\right)+z^{\prime}(c)+$ $z^{\prime}\left(N_{G^{\prime}}^{-}(c)\right)-\beta+[-\gamma]^{+} \leq w^{\prime}(c)+\alpha-\beta-\gamma=w(a)+w(c)+\alpha-\beta-\gamma=w(c)$.

Combining the above observations, we conclude that $\boldsymbol{x}^{*}$ and $\left(\boldsymbol{y}^{*}, \boldsymbol{z}^{*}\right)$ are integral feasible solutions to $\mathbb{P}(G, \boldsymbol{w})$ and $\mathbb{D}(G, \boldsymbol{w})$, respectively. By the above definition, we have $\boldsymbol{w}^{T} \boldsymbol{x}^{*}=\left(\boldsymbol{w}^{\prime}\right)^{T} \boldsymbol{x}^{\prime}=$ $-y^{\prime}\left(A^{\prime}\right)+z^{\prime}\left(V^{\prime}\right)=-y^{*}(A)+y^{*}((a, b))+[\gamma]^{+}+z^{*}(V)-z^{*}(a)-[-\gamma]^{+}=-y^{*}(A)+z^{*}(V)$. From the LP-duality theorem, we further deduce that $\boldsymbol{x}^{*}$ and $\left(\boldsymbol{y}^{*}, \boldsymbol{z}^{*}\right)$ are optimal solutions to $\mathbb{P}(G, \boldsymbol{w})$ and $\mathbb{D}(G, \boldsymbol{w})$, respectively. Hence both (ii) and (iii) follow.

\section{Reduction 3}

Instance: A permissible digraph $G=(V, A)$ with a weight function $\boldsymbol{w} \in \mathbb{Z}^{V}$, and an induced cycle $a b c d a$ of length four in $G$ with $N_{G}^{+}(b)=\{c\}$ and $N_{G}^{+}(d)=\{a\}$. (We remark that $N_{G}^{-}(a) \cap$ $N_{G}^{-}(c)=\emptyset$, otherwise such a common neighbor together with abcda would form a gear.)

Description: Let $G^{\prime}=\left(V^{\prime}, A^{\prime}\right)$ be the digraph obtained from $G$ by identifying $a$ with $c$ (we still use $a$ denote the resulting vertex), and let $w^{\prime}(a)=w(a)+w(c)$ and $w^{\prime}(v)=w(v)$ if $v \in V^{\prime} \backslash a$. Suppose $\boldsymbol{x}^{\prime}$ and $\left(\boldsymbol{y}^{\prime}, \boldsymbol{z}^{\prime}\right)$ are integral optimal solutions to $\mathbb{P}\left(G^{\prime}, \boldsymbol{w}^{\prime}\right)$ and $\mathbb{D}\left(G^{\prime}, \boldsymbol{w}^{\prime}\right)$, respectively. We may assume that $z^{\prime}(a)=0$ (otherwise, replace $z^{\prime}(b)$ by $z^{\prime}(b)+z^{\prime}(a)$ and replace $z^{\prime}(a)$ by 0$)$. As $\phi_{a}\left(\boldsymbol{y}^{\prime}, \boldsymbol{z}^{\prime}\right) \leq w(a)+w(c)$, the above remark implies that one of the inequalities $-\sum_{a \in e} y^{\prime}(e)+z^{\prime}\left(N_{G}^{-}(a)\right) \leq w(a)$ and $-\sum_{c \in e} y^{\prime}(e)+z^{\prime}\left(N_{G}^{-}(c)\right) \leq w(c)$ holds; symmetry allows us to assume the former. Set $\alpha=\left[-\sum_{c \in e} y^{\prime}(e)+z^{\prime}\left(N_{G}^{-}(c)\right)-w(c)\right]^{+}$. Define

- $x^{*}(a)=x^{*}(c)=x^{\prime}(a)$, and $x^{*}(v)=x^{\prime}(v)$ for each $v \in V \backslash\{a, c\}$;

- $y^{*}((c, d))=y^{\prime}((c, d))+\alpha$, and $y^{*}(e)=y^{\prime}(e)$ for each $e \in A \backslash(c, d)$;

- $z^{*}(a)=z^{*}(c)=0, z^{*}(d)=z^{\prime}(d)+\alpha$, and $z^{*}(v)=z^{\prime}(v)$ for each $v \in V \backslash\{a, c, d\}$.

Lemma 5.3. For Reduction 3, the following statements hold:

(i) $G^{\prime}$ is also permissible;

(ii) $\boldsymbol{x}^{*}$ and $\left(\boldsymbol{y}^{*}, \boldsymbol{z}^{*}\right)$ are integral optimal solutions to $\mathbb{P}(G, \boldsymbol{w})$ and $\mathbb{D}(G, \boldsymbol{w})$, respectively; and

(iii) the optimal value of $\mathbb{P}(G, \boldsymbol{w})$ equals that of $\mathbb{P}\left(G^{\prime}, \boldsymbol{w}^{\prime}\right)$. 
Proof. Suppose on the contrary that $G^{\prime}$ contains a subgraph $\Sigma$ which is isomorphic to an odd cycle, or a gear, or a ring. It is then a routine matter to check that the subgraph of $G$ induced by all arcs in $\Sigma \cup\{(a, b),(b, c),(c, d),(d, a)\}$ would contain an odd cycle, or a gear, or a ring, contradicting the hypothesis that $G$ is permissible. So (i) holds.

Clearly $\boldsymbol{x}^{*}$ is an integral feasible solution to $\mathbb{P}(G, \boldsymbol{w})$. Let us now show that $\phi_{v}\left(\boldsymbol{y}^{*}, \boldsymbol{z}^{*}\right) \leq w(v)$ for all $v \in V$. Since $N_{G}^{+}(b)=\{c\}$ and $N_{G}^{+}(d)=\{a\}$, we have $\phi_{v}\left(\boldsymbol{y}^{*}, \boldsymbol{z}^{*}\right)=\phi_{v}\left(\boldsymbol{y}^{\prime}, \boldsymbol{z}^{\prime}\right) \leq w(v)$ for each $v \in V \backslash\{a, c, d\}$. From the above definition, we see that

- $\phi_{d}\left(\boldsymbol{y}^{*}, \boldsymbol{z}^{*}\right)=\phi_{d}\left(\boldsymbol{y}^{\prime}, \boldsymbol{z}^{\prime}\right) \leq w(d)$;

- $\phi_{c}\left(\boldsymbol{y}^{*}, \boldsymbol{z}^{*}\right)=-\sum_{c \in e} y^{\prime}(e)+z^{\prime}\left(N_{G}^{-}(c)\right)-\alpha=\left[-\sum_{c \in e} y^{\prime}(e)+z^{\prime}\left(N_{G}^{-}(c)\right)-w(c)\right]-\alpha+w(c) \leq$ $w(c)$; and

- $\phi_{a}\left(\boldsymbol{y}^{*}, \boldsymbol{z}^{*}\right)=-\sum_{a \in e} y^{\prime}(e)+z^{\prime}\left(N_{G}^{-}(a)\right)+\alpha \leq \max \left\{w(a),-\sum_{a \in e} y^{\prime}(e)+z^{\prime}\left(N_{G}^{-}(a)\right)+\right.$ $\left.\left(-\sum_{c \in e} y^{\prime}(e)+z^{\prime}\left(N_{G}^{-}(c)\right)-w(c)\right)\right\}=\max \left\{w(a), \phi_{a}\left(\boldsymbol{y}^{\prime}, \boldsymbol{z}^{\prime}\right)-w(c)\right\}=w(a)$.

Combining the above observations, we conclude that $\left(\boldsymbol{y}^{*}, \boldsymbol{z}^{*}\right)$ is an integral feasible solution to $\mathbb{D}(G, \boldsymbol{w})$. Since $\boldsymbol{w}^{T} \boldsymbol{x}^{*}=\left(\boldsymbol{w}^{\prime}\right)^{T} \boldsymbol{x}^{\prime}=-y^{\prime}\left(A^{\prime}\right)+z^{\prime}\left(V^{\prime}\right)=-y^{*}(A)+z^{*}(V)$, from the LP-duality theorem, we deduce that $\boldsymbol{x}^{*}$ and $\left(\boldsymbol{y}^{*}, \boldsymbol{z}^{*}\right)$ are optimal solutions to $\mathbb{P}(G, \boldsymbol{w})$ and $\mathbb{D}(G, \boldsymbol{w})$, respectively. Hence both (ii) and (iii) are established.

\section{Reduction 4}

Instance: A permissible digraph $G=(V, A)$ with a weight function $\boldsymbol{w} \in \mathbb{Z}^{V}$, and a path $a b c$ of $G$ with $(b, a) \in A$ and $N_{G}^{+}(a)=\{b\}$ (arc $(c, b)$ may exist).

Lemma 5.4. $\mathbb{P}(G, \boldsymbol{w})$ has an integral optimal solution.

Proof. Let $\boldsymbol{x}$ be an optimal solution to $\mathbb{P}(G, \boldsymbol{w})$. We propose to construct an integral optimal solution $\boldsymbol{x}^{*}$ to $\mathbb{P}(G, \boldsymbol{w})$, starting from $\boldsymbol{x}$.

By (1.3) and (1.4), we have $x(a)+x(b)=1$, which implies $\boldsymbol{x} \in F K(G \backslash(b, c))$. Since $G \backslash(b, c)$ is kernel ideal (recall the assumption at the beginning of this section), $\boldsymbol{x}=\sum_{i=1}^{k} c_{i} \boldsymbol{x}_{i}$, where $\boldsymbol{x}_{i}$ is the incidence vector of a kernel $U_{i}$ in $G \backslash(b, c), c_{i}>0$, for $1 \leq i \leq k$, and $\sum_{i=1}^{k} c_{i}=1$. Renaming subscripts if necessary, we may assume that $\boldsymbol{w}^{T} \boldsymbol{x}_{1} \leq \boldsymbol{w}^{T} \boldsymbol{x}_{i}$ for all $2 \leq i \leq k$. Thus $\{b, c\} \subseteq U_{1}$, for otherwise $\boldsymbol{x}^{*}=\boldsymbol{x}_{1}$ is as desired. It follows that $x_{1}(b)=x_{1}(c)=1$.

Since $x(b)+x(c) \leq 1$ and $c_{1} x_{1}(v) \leq x(v)$ for $v=b, c$, we obtain $c_{1} \leq \min \{x(b), x(c)\} \leq$ $1 / 2$. Let $\alpha$ be the constant in $\left[c_{1}, 1 / 2\right]$ such that $\frac{1-\alpha}{1-c_{1}}\left(x(b)+x(c)-2 c_{1}\right)+2 \alpha=1$, and set $\boldsymbol{x}^{\prime}=\alpha \boldsymbol{x}_{1}+\frac{1-\alpha}{1-c_{1}} \sum_{i=2}^{k} c_{i} \boldsymbol{x}_{i}$. Clearly, $\boldsymbol{x}^{\prime} \in K(G \backslash(b, c))$ and $\boldsymbol{w}^{T} \boldsymbol{x}^{\prime} \leq \boldsymbol{w}^{T} \boldsymbol{x}$. Since $x^{\prime}(b)+x^{\prime}(c)=$ $\frac{1-\alpha}{1-c_{1}}\left(x(b)+x(c)-2 c_{1}\right)+2 \alpha=1$, from the definition we deduce that $\boldsymbol{x}^{\prime} \in F K(G \backslash(b, a))$. As $G \backslash(b, a)$ is kernel ideal, we further have $\boldsymbol{x}^{\prime} \in K(G \backslash(b, a))$. Therefore there exists the incidence vector $\boldsymbol{x}^{*}$ of a kernel $U$ of $G \backslash(b, a)$ such that $\boldsymbol{w}^{T} \boldsymbol{x}^{*} \leq \boldsymbol{w}^{T} \boldsymbol{x}^{\prime}$; this $\boldsymbol{x}^{*}$ is as desired because $U$ is also a kernel of $G$ and $\boldsymbol{w}^{T} \boldsymbol{x}^{*} \leq \boldsymbol{w}^{T} \boldsymbol{x}$.

In the remainder of this reduction, we reserve the symbol $\boldsymbol{x}$ for an integral optimal solution to $\mathbb{P}(G, \boldsymbol{w})$ and the pair $(\boldsymbol{y}, \boldsymbol{z})$ for an optimal solution to $\mathbb{P}(G, \boldsymbol{w})$. Set $\delta=y((b, c))$.

Description: Let $G^{\prime}=(V, A \backslash(b, c))$, and let $w^{\prime}(v)=w(v)+\lceil\delta\rceil$ if $v \in\{b, c\}$ and $w^{\prime}(v)=w(v)$ if $v \in V \backslash\{b, c\}$. Suppose $\boldsymbol{x}^{\prime}$ and $\left(\boldsymbol{y}^{\prime}, \boldsymbol{z}^{\prime}\right)$ are integral optimal solutions to $\mathbb{P}\left(G^{\prime}, \boldsymbol{w}^{\prime}\right)$ and $\mathbb{D}\left(G^{\prime}, \boldsymbol{w}^{\prime}\right)$, respectively. In particular, set $\boldsymbol{x}^{\prime}=\boldsymbol{x}$ if $\boldsymbol{x}$ is also an optimal solution to $\mathbb{P}\left(G^{\prime}, \boldsymbol{w}^{\prime}\right)$. Since 
$N_{G}^{+}(a)=\{b\}$, we may assume that $z^{\prime}(b)=0$ (otherwise we replace $z^{\prime}(a)$ by $z^{\prime}(a)+z^{\prime}(b)$ and replace $z^{\prime}(b)$ by 0$)$. Define

- $x^{*}=x^{\prime}$

- $y^{*}((b, c))=\lceil\delta\rceil$ and $y^{*}(e)=y^{\prime}(e)$ for all $e \in A \backslash(b, c)$; and

- $z^{*}=z^{\prime}$.

Lemma 5.5. For Reduction 4, the following statements hold:

(i) $G^{\prime}$ is also permissible;

(ii) $\boldsymbol{x}^{*}$ and $\left(\boldsymbol{y}^{*}, \boldsymbol{z}^{*}\right)$ are integral optimal solutions to $\mathbb{P}(G, \boldsymbol{w})$ and $\mathbb{D}(G, \boldsymbol{w})$, respectively; and

(iii) the optimal value of $\mathbb{P}(G, \boldsymbol{w})$ equals that of $\mathbb{P}\left(G^{\prime}, \boldsymbol{w}^{\prime}\right)$ minus $\lceil\delta\rceil$.

Proof. Statement (i) is trivial. Define $w^{\prime \prime}(v)=w(v)+\delta$ if $v \in\{b, c\}$ and $w^{\prime \prime}(v)=w(v)$ if $v \in V \backslash\{b, c\}$. Clearly, $\boldsymbol{w}^{\prime}=\boldsymbol{w}^{\prime \prime}$ if $\delta$ is integral. Observe that $\boldsymbol{x}$ and $\left(\left.\boldsymbol{y}\right|_{A \backslash(b, c)}, \boldsymbol{z}\right)$ are feasible solutions to $\mathbb{P}\left(G^{\prime}, \boldsymbol{w}^{\prime \prime}\right)$ and $\mathbb{D}\left(G^{\prime}, \boldsymbol{w}^{\prime \prime}\right)$, respectively, and satisfy the complementary slackness condition. So

(1) $\boldsymbol{x}$ is also an optimal solution to $\mathbb{P}\left(G^{\prime}, \boldsymbol{w}^{\prime \prime}\right)$ and hence $\left(\boldsymbol{w}^{\prime \prime}\right)^{T} \boldsymbol{x}^{\prime} \geq\left(\boldsymbol{w}^{\prime \prime}\right)^{T} \boldsymbol{x}$.

Let us first consider the case when $\boldsymbol{x}$ is an optimal solution to $\mathbb{P}\left(G^{\prime}, \boldsymbol{w}^{\prime}\right)$. Now $\boldsymbol{x}^{*}=\boldsymbol{x}$ and $\left(\boldsymbol{w}^{\prime}\right)^{T} \boldsymbol{x}=-y^{\prime}(A \backslash(b, c))+z^{\prime}(V)$. So $\phi_{b}\left(\boldsymbol{y}^{*}, \boldsymbol{z}^{*}\right)=\phi_{b}\left(\boldsymbol{y}^{\prime}, \boldsymbol{z}^{\prime}\right)-y^{*}((b, c)) \leq w^{\prime}(b)-\lceil\delta\rceil=w(b)$. As $z^{\prime}(b)=0$, we have $\phi_{c}\left(\boldsymbol{y}^{*}, \boldsymbol{z}^{*}\right)=\phi_{c}\left(\boldsymbol{y}^{\prime}, \boldsymbol{z}^{\prime}\right)-y^{*}((b, c))+z^{\prime}(b) \leq w^{\prime}(c)-\lceil\delta\rceil=w(c)$. From the feasibility of $\left(\boldsymbol{y}^{\prime}, \boldsymbol{z}^{\prime}\right)$ we deduce that $\left(\boldsymbol{y}^{*}, \boldsymbol{z}^{*}\right)$ is a feasible solution to $\mathbb{D}(G, \boldsymbol{w})$, with value $-y^{*}(A)+z^{*}(V)=-y^{*}((b, c))-y^{\prime}(A \backslash(b, c))+z^{\prime}(V)=\left(\boldsymbol{w}^{\prime}\right)^{T} \boldsymbol{x}-\lceil\delta\rceil$. Again, by the complementary slackness condition, $\delta(x(b)+x(c)-1)=0$, so either $x(b)+x(c)=1$ or $\delta=x(b)=x(c)=0$ (as $\boldsymbol{x}$ is integral), and hence the equality $\boldsymbol{w}^{T} \boldsymbol{x}=\left(\boldsymbol{w}^{\prime}\right)^{T} \boldsymbol{x}-\lceil\delta\rceil$ holds in either subcase. Therefore $-y^{*}(A)+z^{*}(V)=\boldsymbol{w}^{T} \boldsymbol{x}$. From the LP-duality theorem, we deduce that $\left(\boldsymbol{y}^{*}, \boldsymbol{z}^{*}\right)$ is an integral optimal solution to $\mathbb{D}(G, \boldsymbol{w})$.

So we assume that $\boldsymbol{x}$ is not an optimal solution to $\mathbb{P}\left(G^{\prime}, \boldsymbol{w}^{\prime}\right)$. Thus $\boldsymbol{w}^{\prime} \neq \boldsymbol{w}^{\prime \prime}$ by (1). It follows that $\delta$ is not integral. By the complementary slackness condition, we get

(2) $x(b)+x(c)=1$.

Let $\omega^{*}$ denote the optimal value of $\mathbb{P}(G, \boldsymbol{w})$ (which is an integer as $\boldsymbol{x}$ is integral). From (2) we see that

(3) $\left(\boldsymbol{w}^{\prime \prime}\right)^{T} \boldsymbol{x}=\omega^{*}+\delta$ and $\left(\boldsymbol{w}^{\prime}\right)^{T} \boldsymbol{x}=\omega^{*}+\lceil\delta\rceil$.

Since $\boldsymbol{x}$ is not an optimal solution to $\mathbb{P}\left(G^{\prime}, \boldsymbol{w}^{\prime}\right)$, we have $\left(\boldsymbol{w}^{\prime}\right)^{T} \boldsymbol{x}^{\prime}<\left(\boldsymbol{w}^{\prime}\right)^{T} \boldsymbol{x}=\omega^{*}+\lceil\delta\rceil$. So

(4) $\left(\boldsymbol{w}^{\prime}\right)^{T} \boldsymbol{x}^{\prime} \leq \omega^{*}+\lceil\delta\rceil-1$.

From the definitions of $\boldsymbol{w}^{\prime}$ and $\boldsymbol{w}^{\prime \prime}$, we see that $\left(\boldsymbol{w}^{\prime}\right)^{T} \boldsymbol{x}^{\prime} \geq\left(\boldsymbol{w}^{\prime \prime}\right)^{T} \boldsymbol{x}^{\prime}$. Using (1) and (3), we thus obtain $\left(\boldsymbol{w}^{\prime}\right)^{T} \boldsymbol{x}^{\prime} \geq\left(\boldsymbol{w}^{\prime \prime}\right)^{T} \boldsymbol{x}=\omega^{*}+\delta$, contradicting (4).

\section{Reduction 5}

Instance: A permissible digraph $G=(V, A)$ with a weight function $\boldsymbol{w} \in \mathbb{Z}^{V}$, and three distinct vertices $a, b, c$ of $G$, such that $N_{G}^{+}(a)=\{b\}, N_{G}^{+}(b)=\{a\}$, and $(c, b) \in A$, and that $G \backslash(c, b)$ is the union of two disjoint graphs $G_{1}=\left(V_{1}, A_{1}\right)$ and $G \backslash V_{1}$, with $\{a, b\} \varsubsetneqq V_{1}$ and $c \notin V_{1}$.

Description: Let $G_{2}=\left(V_{2}, A_{2}\right)$ denote $G \backslash\left(V_{1} \backslash\{a, b\}\right)$, let $\alpha$ be the minimum weight of a kernel in $\left(G_{2} \backslash\{a, b\},\left.\boldsymbol{w}\right|_{V_{2} \backslash\{a, b\}}\right)$, and let $\beta$ be the minimum weight of a kernel in $\left(G_{2} \backslash\{a, b, c\},\left.\boldsymbol{w}\right|_{V_{2} \backslash\{a, b, c\}}\right)$ if $V_{2} \neq\{a, b, c\}$ and let $\beta=0$ otherwise. Define $w_{1}(a)=w(a)+\alpha, w_{1}(b)=w(b)+\beta$, 
and $w_{1}(v)=w(v)$ if $v \in V_{1} \backslash\{a, b\}$, and define $w_{2}(a)=-\alpha, w_{2}(b)=-\beta$, and $w_{2}(v)=v$ if $v \in V_{2} \backslash\{a, b\}$. Observe that the minimum weight of a kernel in $\left(G_{2}, \boldsymbol{w}_{2}\right)$ is zero, and that each of $a$ and $b$ is contained in a minimum weighted kernel of $\left(G_{2}, \boldsymbol{w}_{2}\right)$. Suppose $\boldsymbol{x}_{i}$ and $\left(\boldsymbol{y}_{i}, \boldsymbol{z}_{i}\right)$ are integral optimal solutions to $\mathbb{P}\left(G_{i}, \boldsymbol{w}_{i}\right)$ and $\mathbb{D}\left(G_{i}, w_{i}\right)$, respectively, for $i=1,2$, such that $x_{1}(v)=x_{2}(v)$ for $v \in\{a, b\}$. (Given $\boldsymbol{x}_{1}$, the existence of such an $\boldsymbol{x}_{2}$ is guaranteed by the preceding observation.) Define

- $x^{*}(v)=x_{i}(v)$ if $v \in V_{i}$ for $i=1,2$;

- $y^{*}(e)=y_{1}(e)+y_{2}(e)$ if $e \in\{(a, b),(b, a)\}$, and $y^{*}(e)=y_{i}(e)$ if $e \in A_{i} \backslash\{(a, b),(b, a)\}$ for $i=1,2 ;$ and

- $z^{*}(v)=z_{1}(v)+z_{2}(v)$ if $v \in\{a, b\}$, and $z^{*}(v)=z_{i}(v)$ if $v \in V_{i} \backslash\{a, b\}$ for $i=1,2$.

Lemma 5.6. For Reduction 5, the following statements hold:

(i) $\boldsymbol{x}^{*}$ and $\left(\boldsymbol{y}^{*}, \boldsymbol{z}^{*}\right)$ are integral optimal solutions to $\mathbb{P}(G, \boldsymbol{w})$ and $\mathbb{D}(G, \boldsymbol{w})$, respectively; and

(ii) the optimal value of $\mathbb{P}(G, \boldsymbol{w})$ equals that of $\mathbb{P}\left(G_{1}, \boldsymbol{w}_{1}\right)$.

Proof. (i) From its definition, we see that $\boldsymbol{x}^{*}$ is a feasible solution to $\mathbb{P}(G, \boldsymbol{w})$. To establish the dual feasibility of $\left(\boldsymbol{y}^{*}, \boldsymbol{z}^{*}\right)$, it suffices to verify that $\phi_{v}\left(\boldsymbol{y}^{*}, \boldsymbol{z}^{*}\right) \leq w(v)$ for $v \in$ $\{a, b\}$. Indeed, by definition, $\phi_{v}\left(\boldsymbol{y}^{*}, \boldsymbol{z}^{*}\right)=-\sum_{v \in e \in A} y^{*}(e)+z^{*}(a)+z^{*}(b)+z^{*}\left(N_{G}^{-}(v) \backslash\{a, b\}\right)=$ $\sum_{i=1}^{2}\left[-\sum_{v \in e \in A_{i} \backslash\{(a, b),(b, a)\}} y_{i}(e)-y_{i}((a, b))-y_{i}((b, a))+z_{i}(a)+z_{i}(b)+z_{i}\left(N_{G_{i}}^{-}(v) \backslash\{a, b\}\right)\right]=$ $\sum_{i=1}^{2}\left[-\sum_{v \in e \in A_{i}} y_{i}(e)+z_{i}(v)+z_{i}\left(N_{G_{i}}^{-}(v)\right)\right] \leq w_{1}(v)+w_{2}(v)=w(v)$, so $\left(\boldsymbol{y}^{*}, \boldsymbol{z}^{*}\right)$ is a feasible solution to $\mathbb{D}(G, \boldsymbol{w})$. As $w_{1}(v)+w_{2}(v)=w(v)$ and $x_{1}(v)=x_{2}(v)$ for $v \in\{a, b\}$, we have $\boldsymbol{w}^{T} \boldsymbol{x}^{*}=\sum_{i=1}^{2} \boldsymbol{w}_{i}^{T} \boldsymbol{x}_{i}=\sum_{i=1}^{2}\left[-y_{i}\left(A_{i}\right)+z_{i}\left(V_{i}\right)\right]=-y^{*}(A)+z^{*}(V)$. By the LP-duality theorem, $\boldsymbol{x}^{*}$ and $\left(\boldsymbol{y}^{*}, \boldsymbol{z}^{*}\right)$ are integral optimal solutions to $\mathbb{P}(G, \boldsymbol{w})$ and $\mathbb{D}(G, \boldsymbol{w})$, respectively.

(ii) Since the minimum weight of a kernel in $\left(G_{2}, \boldsymbol{w}_{2}\right)$ is zero (see description above), $\boldsymbol{w}_{2}^{T} \boldsymbol{x}_{2}=0$. So $\boldsymbol{w}^{T} \boldsymbol{x}^{*}=\sum_{i=1}^{2} \boldsymbol{w}_{i}^{T} \boldsymbol{x}_{i}=\boldsymbol{w}_{1}^{T} \boldsymbol{x}_{1}$. Hence the optimal value of $\mathbb{P}(G, \boldsymbol{w})$ equals that of $\mathbb{P}\left(G_{1}, \boldsymbol{w}_{1}\right)$.

\section{Reduction 6}

Instance: A permissible digraph $G=(V, A)$ with a weight function $\boldsymbol{w} \in \mathbb{Z}^{V}$, and three distinct vertices $a, b, c$ of $G$, such that $N_{G}^{+}(a)=N_{G}^{-}(a)=\{b\}, N_{G}^{+}(b)=\{a\}$, and $N_{G}^{-}(b)=\{a, c\}$.

Throughout this reduction, $G^{\prime}=\left(V^{\prime}, A^{\prime}\right)$ stands for the digraph arising from $G \backslash a$ by adding an $\operatorname{arc}(b, c)$. Clearly, $G^{\prime}$ is permissible and hence is both kernel ideal and kernel Mengerian by the assumption at the beginning of this section. Let $\omega^{*}$ stand for the common optimal value of $\mathbb{P}(G, \boldsymbol{w})$ and $\mathbb{D}(G, \boldsymbol{w})$. For each subgraph $H$ of $G$, we use $\Gamma(H)$ to denote the set of all kernels in $H$, and use $\chi_{U} \in\{0,1\}^{V(H)}$ to denote the incidence vector of each $U$ in $\Gamma(H)$.

Lemma 5.7. $\mathbb{P}(G, \boldsymbol{w})$ has an integral optimal solution.

Proof. Let $\boldsymbol{x}$ be an optimal solution to $\mathbb{P}(G, \boldsymbol{w})$ such that

(1) $x(b)$ is as large as possible.

By (1.3) and (1.4), we have

(2) $x(a)+x(b)=1$.

Suppose for a contradiction that $\boldsymbol{x}$ is not integral. Let us make some further observations about $\boldsymbol{x}$. 
(3) $x(b)+x(c)<1$.

Otherwise, $x(b)+x(c)=1$ by (1.3). Thus $x(a)=x(c)$ by (2) and $\left.\boldsymbol{x}\right|_{V^{\prime}} \in F K\left(G^{\prime}\right)$. Let $w^{\prime}(c)=w(a)+w(c)$ and $w^{\prime}(v)=w(v)$ for every $v \in V^{\prime} \backslash c$. As $G^{\prime}$ is kernel ideal, $\mathbb{P}\left(G^{\prime}, \boldsymbol{w}^{\prime}\right)$ admits an integral optimal solution $\boldsymbol{x}^{\prime}$, with value $\left(\boldsymbol{w}^{\prime}\right)^{T} \boldsymbol{x}^{\prime} \leq\left.\left(\boldsymbol{w}^{\prime}\right)^{T} \boldsymbol{x}\right|_{V^{\prime}}=\boldsymbol{w}^{T} \boldsymbol{x}-w(a) x(a)-$ $w(c) x(c)+w^{\prime}(c) x(c)=\boldsymbol{w}^{T} \boldsymbol{x}=\omega^{*}$. Define $\boldsymbol{x}^{*} \in \mathbb{Z}_{+}^{V}$ by $x^{*}(a)=x^{\prime}(c)$ and $x^{*}(v)=x^{\prime}(v)$ for every $v \in V^{\prime}$. Clearly, $\boldsymbol{x}^{*}$ is a feasible solution to $\mathbb{P}(G, \boldsymbol{w})$ with value $\boldsymbol{w}^{T} \boldsymbol{x}^{*}=\left(\boldsymbol{w}^{\prime}\right)^{T} \boldsymbol{x}^{\prime} \leq \omega^{*}$. So $\boldsymbol{x}^{*}$ is an integral optimal solution to $\mathbb{P}(G, \boldsymbol{w})$. Hence we may assume that (3) holds.

(4) $0<x(a), x(b)<1$.

Otherwise, $x(u)=1$ for some $u \in\{a, b\}$. Let $e \in\{(a, b),(b, a)\}$ be the arc leaving $u$. Then $\boldsymbol{x} \in F K(G \backslash e)$. Since $G \backslash e$ is kernel ideal, $\mathbb{P}(G \backslash e, \boldsymbol{w})$ has an integral optimal solution $\boldsymbol{x}^{*}$, for which $\boldsymbol{w}^{T} \boldsymbol{x}^{*} \leq \boldsymbol{w}^{T} \boldsymbol{x}=\omega^{*}$. Clearly, $\boldsymbol{x}^{*} \in K(G)$. So $\boldsymbol{x}^{*}$ is an optimal integral solution to $\mathbb{P}(G, \boldsymbol{w})$. Hence we may assume (4).

(5) $w(b)>w(a)$.

To justify this, let $\tilde{\boldsymbol{x}}$ be defined by $\tilde{x}(a)=x(c), \tilde{x}(b)=1-x(c)$, and $\tilde{x}(v)=x(v)$ for each $v \in V \backslash\{a, b\}$. Then $\tilde{\boldsymbol{x}}$ is a feasible solution to $\mathbb{P}(G, \boldsymbol{x})$. From (1) and (3), we deduce that $\boldsymbol{w}^{T} \tilde{\boldsymbol{x}}>$ $\boldsymbol{w}^{T} \boldsymbol{x}$, which implies $w(a) x(c)+w(b)(1-x(c))=w(a) \tilde{x}(a)+w(b) \tilde{x}(b)>w(a) x(a)+w(b) x(b)$, so $(1-x(b)-x(c)) w(b)>(x(a)-x(c)) w(a)$ and hence $w(b)>w(a)$ by (2) and (3).

Let $(\boldsymbol{y}, \boldsymbol{z})$ be an optimal solution to $\mathbb{D}(G, \boldsymbol{w})$ with minimum $z(c)$. By the complementary slackness condition and (4), we obtain

(6) $\phi_{v}(\boldsymbol{y}, \boldsymbol{z})=w(v)$ for $v \in\{a, b\}$.

(7) $y((c, b))=0, z(c)=w(b)-w(a)>0$, and $x(c)+x\left(N_{G}^{+}(c)\right)=1$.

To justify this, note that $\phi_{b}(\boldsymbol{y}, \boldsymbol{z})=\phi_{a}(\boldsymbol{y}, \boldsymbol{z})-y((c, b))+z(c)$. So $-y((c, b))+z(c)=$ $w(b)-w(a)>0$ by (6) and (5). Hence $y((c, b))=0$ and $z(c)=w(b)-w(a)>0$, for otherwise, let $(\overline{\boldsymbol{y}}, \overline{\boldsymbol{z}})$ be obtained from $(\boldsymbol{y}, \boldsymbol{z})$ by replacing $z(c)$ with $z(c)-y((c, b))$ and $y((c, b))$ with 0 . Then $(\overline{\boldsymbol{y}}, \overline{\boldsymbol{z}})$ is also an optimal solution to $\mathbb{D}(G, \boldsymbol{w})$ with $\bar{z}(c)<z(c)$, contradicting the minimality assumption on $(\boldsymbol{y}, \boldsymbol{z})$. Since $z(c)>0$, from the complementary slackness condition we deduce that $x(c)+x\left(N_{G}^{+}(c)\right)=1$. So $(7)$ is established.

Let $\boldsymbol{x}^{\prime} \in \mathbb{R}_{+}^{V^{\prime}}$ be defined by $x^{\prime}(b)=1-x(c)$ and $x^{\prime}(v)=x(v)$ if $v \in V^{\prime} \backslash b$. Then $\boldsymbol{x}^{\prime} \in F K\left(G^{\prime}\right)$. Since $G^{\prime}$ is kernel ideal, $\boldsymbol{x}^{\prime}=\sum_{U \in \Gamma\left(G^{\prime}\right)} r_{U} \boldsymbol{\chi}_{U}$, where $\sum_{U \in \Gamma\left(G^{\prime}\right)} r_{U}=1$ and $r_{U} \geq 0$ for all $U \in \Gamma\left(G^{\prime}\right)$. Set $\mathcal{D}=\left\{U \in \Gamma\left(G^{\prime}\right): r_{U}>0\right\}$. Clearly, $\mathcal{D}$ is the disjoint union of the following three sets:

- $\mathcal{A}=\left\{U \in \mathcal{D}: b \in U, U \backslash b \in \Gamma\left(G^{\prime} \backslash b\right)\right\} ;$

- $\mathcal{B}=\left\{U \in \mathcal{D}: b \in U, U \backslash b \notin \Gamma\left(G^{\prime} \backslash b\right)\right\} ;$ and

- $\mathcal{C}=\{U \in \mathcal{D}: c \in U\}$.

We propose to show that

(8) $\mathcal{A} \neq \emptyset \neq \mathcal{B}$.

Indeed, by (7), we have $x(b)+x(c)+x\left(N_{G^{\prime}}^{+}(c) \backslash b\right)=x(c)+x\left(N_{G}^{+}(c)\right)=1$. Using (3), we obtain $x\left(N_{G^{\prime}}^{+}(c) \backslash b\right)>0$. So $x^{\prime}\left(N_{G^{\prime}}^{+}(c) \backslash b\right)>0$ and hence $\mathcal{A} \neq \emptyset$. Suppose on the contrary that $\mathcal{B}=\emptyset$. Then $\boldsymbol{x}^{\prime}=\sum_{U \in \mathcal{A}} r_{U} \boldsymbol{\chi}_{U}+\sum_{U \in \mathcal{C}} r_{U} \boldsymbol{\chi}_{U}$. Define $\boldsymbol{x}^{*}=\sum_{U \in \mathcal{A}} r_{U} \chi_{\{a\} \cup(U \backslash b)}+\sum_{U \in \mathcal{C}} r_{U} \boldsymbol{\chi}_{\{a\} \cup U}$. From the definitions of $\mathcal{A}$ and $\mathcal{C}$, we see that $\boldsymbol{x}^{*} \in F K(G)$. Note that $x^{*}(a)=x^{\prime}(b)+x^{\prime}(c)=$ $1-x(c)+x(c)=1, x^{*}(b)=0$, and $x^{*}(c)=x^{\prime}(c)=x(c)$. So $\boldsymbol{w}^{T} \boldsymbol{x}^{*}-\boldsymbol{w}^{T} \boldsymbol{x}=w(a) x^{*}(a)-$ $w(a) x(a)-w(b) x(b)<w(a)-w(a) x(a)-w(a) x(b)=w(a)-w(a)=0$, where the inequality follows from (5) and the first equality from (2). Thus $\boldsymbol{w}^{T} \boldsymbol{x}^{*}<\boldsymbol{w}^{T} \boldsymbol{x}=\omega^{*}$; this contradiction implies that $\mathcal{B} \neq \emptyset$. 
Take $A$ in $\mathcal{A}$ and $B$ in $\mathcal{B}$. From the definitions of $\mathcal{A}$ and $\mathcal{B}$, we see that

(9) $b \in A \cap B, c \notin A \cup B$, and $A \cap\left(N_{G^{\prime}}^{+}(c) \backslash b\right) \neq \emptyset=B \cap\left(N_{G^{\prime}}^{+}(c) \backslash b\right)$.

Let $\theta=\left|A \cap\left(N_{G^{\prime}}^{+}(c) \backslash b\right)\right|$. Then $\theta \geq 1$ by (9). Set $\alpha=1$ if $w(A \backslash b)+\theta w(a) \geq w(B \backslash b)+\theta w(b)$ and $\alpha=2$ otherwise. In view of (2)-(4), we can find a constant $\epsilon$ such that $0<\epsilon<\min \left\{r_{A}, r_{B}\right\}$ and $0<\theta \epsilon \leq \min \{x(b), 1-x(b)-x(c)\}=\min \{1-x(a), x(a)-x(c)\}$. Let $\boldsymbol{x}^{\prime \prime}=\boldsymbol{x}^{\prime}+\epsilon$. $\left[(-1)^{\alpha} \boldsymbol{\chi}_{A}+(-1)^{3-\alpha} \boldsymbol{\chi}_{B}\right]$. It is clear from $\epsilon<\min \left\{r_{A}, r_{B}\right\}$ that $\boldsymbol{x}^{\prime \prime}$ remains to be a convex combination of incidence vectors of kernels of $G^{\prime}$ in $\mathcal{D}$. So $\boldsymbol{x}^{\prime \prime} \in F K\left(G^{\prime}\right)$. Define $\boldsymbol{x}^{*} \in \mathbb{R}^{V}$ by $x^{*}(a)=x(a)+(-1)^{\alpha} \theta \epsilon, x^{*}(b)=x(b)+(-1)^{3-\alpha} \theta \epsilon$, and $x^{*}(v)=x^{\prime \prime}(v)$ for all $v \in V \backslash\{a, b\}$.

(10) $\boldsymbol{x}^{*} \in F K(G)$.

To justify this, note that $0 \leq x^{*}(a), x^{*}(b) \leq 1$ by the choice of $\epsilon$, and that $x^{*}(c)=x^{\prime \prime}(c)=$ $x^{\prime}(c)=x(c)$ because $c \notin A \cup B$ by (9). Moreover, $x^{*}(a)+x^{*}(b)=x(a)+x(b)=1$ by (2), and $x^{*}(b)+x^{*}(c)=x(b)+x(c)+(-1)^{3-\alpha} \theta \epsilon \leq 1$ by the choice of $\epsilon$. Since $x^{\prime \prime}\left(N_{G^{\prime}}^{+}(c) \backslash b\right)=$ $x^{\prime}\left(N_{G^{\prime}}^{+}(c) \backslash b\right)+(-1)^{\alpha} \theta \epsilon$ by (9) and the definition of $\theta$, we have $x^{*}(c)+x^{*}\left(N_{G}^{+}(c)\right)=x^{*}(b)+x^{*}(c)+$ $x^{*}\left(N_{G}^{+}(c) \backslash b\right)=x(b)+(-1)^{3-\alpha} \theta \epsilon+x^{\prime \prime}(c)+x^{\prime \prime}\left(N_{G^{\prime}}^{+}(c) \backslash b\right)=x(b)+(-1)^{3-\alpha} \theta \epsilon+x^{\prime}(c)+x^{\prime}\left(N_{G^{\prime}}^{+}(c) \backslash b\right)+$ $(-1)^{\alpha} \theta \epsilon=x(b)+x^{\prime}(c)+x^{\prime}\left(N_{G^{\prime}}^{+}(c) \backslash b\right)=x(b)+x(c)+x\left(N_{G}^{+}(c) \backslash b\right)=x(c)+x\left(N_{G}^{+}(c)\right)=1$ by (7). So (10) holds.

(11) $\boldsymbol{w}^{T} \boldsymbol{x}^{*}=\boldsymbol{w}^{T} \boldsymbol{x}+(-1)^{\alpha} \epsilon \cdot[w(A \backslash b)+\theta w(a)-w(B \backslash b)-\theta w(b)]$.

By direct computation, we obtain $\boldsymbol{w}^{T} \boldsymbol{x}^{*}=w(a) x^{*}(a)+w(b) x^{*}(b)+\sum_{v \in V \backslash\{a, b\}} w(v) x^{\prime \prime}(v)=$ $w(a)\left[x(a)+(-1)^{\alpha} \theta \epsilon\right]+w(b)\left[x(b)+(-1)^{3-\alpha} \theta \epsilon\right]+\left[\sum_{v \in V \backslash\{a, b\}} w(v) x^{\prime}(v)+(-1)^{\alpha} w(A \backslash b) \epsilon+(-1)^{3-\alpha}\right.$ $w(B \backslash b) \epsilon]=\left[w(a) x(a)+w(b) x(b)+\sum_{v \in V \backslash\{a, b\}} w(v) x^{\prime}(v)\right]+(-1)^{\alpha} \epsilon \cdot[w(A \backslash b)+\theta w(a)-w(B \backslash b)-$ $\theta w(b)]=\boldsymbol{w}^{T} \boldsymbol{x}+(-1)^{\alpha} \epsilon \cdot[w(A \backslash b)+\theta w(a)-w(B \backslash b)-\theta w(b)]$. So (11) is justified.

(12) $\alpha=1$ and $\boldsymbol{x}^{*}$ is also an optimal solution to $\mathbb{P}(G, \boldsymbol{w})$.

Suppose the contrary: $\alpha=2$. From the definition of $\alpha$, we see that $w(A \backslash b)+\theta w(a)<$ $w(B \backslash b)+\theta w(b)$. Thus $\boldsymbol{w}^{T} \boldsymbol{x}^{*}<\boldsymbol{w}^{T} \boldsymbol{x}=\omega^{*}$ by (11), a contradiction (see (10)). So $\alpha=1$ and hence $w(A \backslash b)+\theta w(a) \geq w(B \backslash b)+\theta w(b)$ by the definition of $\alpha$. It follows from (11) that $\boldsymbol{w}^{T} \boldsymbol{x}^{*} \leq \boldsymbol{w}^{T} \boldsymbol{x}$. Therefore, by $(10), \boldsymbol{x}^{*}$ is also an optimal solution to $\mathbb{P}(G, \boldsymbol{w})$.

From (12) we conclude that $x^{*}(b)=x(b)+\theta \epsilon>x(b)$. This contradiction to (1) proves the present lemma.

By Lemma 5.7, the common optimal value $\omega^{*}$ of $\mathbb{P}(G, \boldsymbol{w})$ and $\mathbb{D}(G, \boldsymbol{w})$ is an integer. It remains to prove that $\mathbb{D}(G, \boldsymbol{w})$ admits an integral solution of value $\omega^{*}$. To this end, we assume, throughout the remainder of this reduction, that

each of $a$ and $b$ is contained in a kernel of $(G, \boldsymbol{w})$ with total weight $\omega^{*}$.

Otherwise, let $\kappa(u)$ be the minimum total weight of a kernel containing a vertex $u$ in $(G, \boldsymbol{w})$, and let $\tilde{\boldsymbol{w}}$ be obtained from $\boldsymbol{w}$ by replacing $w(v)$ with $w(v)-\left(\kappa(v)-\omega^{*}\right)$ for $v=a$ and $b$. Since $\tilde{\boldsymbol{w}} \leq \boldsymbol{w}$, from (1.8)-(1.10) we see that every feasible solution to $\mathbb{D}(G, \tilde{\boldsymbol{w}})$ remains feasible to $\mathbb{D}(G, \boldsymbol{w})$. Hence we may assume that (5.1) holds. We reserve the symbol $\boldsymbol{x}_{v}$ for an integral optimal solution to $\mathbb{P}(G, \boldsymbol{w})$ with $x_{v}(v)=1$ for $v \in\{a, b\}$. It is clear that

$$
x_{b}(a)=x_{b}(c)=0 \text { and }\left.\boldsymbol{x}_{b}\right|_{V^{\prime}} \in K\left(G^{\prime}\right) .
$$

The reduction given below depends on whether $w(a) \geq w(b)$ or not.

Case 1. $w(a) \geq w(b)$. 
Description: Let $\boldsymbol{w}^{\prime} \in \mathbb{Z}^{V^{\prime}}$ be defined by $w^{\prime}(c)=w(a)+w(c)$ and $w^{\prime}(v)=w(v)$ if $v \in V^{\prime} \backslash c$. Suppose $\left(\boldsymbol{y}^{\prime}, \boldsymbol{z}^{\prime}\right)$ is an integral optimal solution to $\mathbb{D}\left(G^{\prime}, \boldsymbol{w}^{\prime}\right)$. We may assume that $y^{\prime}((c, b))=0$ and $z^{\prime}(c)=0$ (otherwise, replace $y^{\prime}((b, c))$ with $y^{\prime}((b, c))+y^{\prime}((c, b)), y^{\prime}((c, b))$ with $0, z^{\prime}(b)$ with $z^{\prime}(b)+z^{\prime}(c)$, and $z^{\prime}(c)$ with 0$)$. Let $\alpha=w(a)+y^{\prime}((b, c))-z^{\prime}(b)$. Define

- $y^{*}((a, b))=0, y^{*}((b, a))=y^{\prime}((b, c)), y^{*}((c, b))=\alpha$, and $y^{*}(e)=y^{\prime}(e)$ for each $e \in$ $A^{\prime} \backslash\{(b, c),(c, b)\}$; and

- $z^{*}(a)=\alpha$ and $z^{*}(v)=z^{\prime}(v)$ for each $v \in V \backslash a$.

Lemma 5.8. For Reduction 6 in Case 1, the following statements hold:

(i) $\left(\boldsymbol{y}^{*}, \boldsymbol{z}^{*}\right)$ is an integral optimal solution to $\mathbb{D}(G, \boldsymbol{w})$; and

(ii) the optimal value of $\mathbb{D}(G, \boldsymbol{w})$ equals that of $\mathbb{D}\left(G^{\prime}, \boldsymbol{w}^{\prime}\right)$.

Proof. Let us first show that

(1) the optimal value of $\mathbb{P}\left(G^{\prime}, \boldsymbol{w}^{\prime}\right)$ is $\omega^{*}$. So $-y^{\prime}\left(A^{\prime}\right)+z^{\prime}\left(V^{\prime}\right)=\omega^{*}$ by duality.

Indeed, for any $\boldsymbol{x}^{\prime \prime} \in K\left(G^{\prime}\right)$, let $x(a)=x^{\prime \prime}(c)$ and $x(v)=x^{\prime \prime}(v)$ if $v \in V^{\prime}$. Then $\boldsymbol{x} \in K(G)$ and $\boldsymbol{w}^{T} \boldsymbol{x}=\left(\boldsymbol{w}^{\prime}\right)^{T} \boldsymbol{x}^{\prime \prime}$. Thus $\omega^{*}$ is a lower bound on the optimal value of $\mathbb{P}\left(G^{\prime}, \boldsymbol{w}^{\prime}\right)$. From (5.2) we see that $\boldsymbol{x}^{\prime}=\left.\boldsymbol{x}_{b}\right|_{V^{\prime}}$ is feasible solution to $\mathbb{P}\left(G^{\prime}, \boldsymbol{w}^{\prime}\right)$. Since $\left(\boldsymbol{w}^{\prime}\right)^{T} \boldsymbol{x}^{\prime}=\boldsymbol{w}^{T} \boldsymbol{x}_{b}=\omega^{*}$, statement (1) holds.

Observe that $-y^{*}(A)+z^{*}(V)=\left(-y^{\prime}\left(A^{\prime} \backslash\{(b, c),(c, b)\}\right)-y^{*}((a, b))-y^{*}((b, a))-y^{*}((c, b))\right)+$ $\left(z^{\prime}\left(V^{\prime}\right)+z^{*}(a)\right)=\left(-y^{\prime}\left(A^{\prime}\right)-\alpha\right)+\left(z^{\prime}\left(V^{\prime}\right)+\alpha\right)=-y^{\prime}\left(A^{\prime}\right)+z^{\prime}\left(V^{\prime}\right)$. Using (1), we obtain

(2) $-y^{*}(A)+z^{*}(V)=\omega^{*}$.

(3) $\left(\boldsymbol{y}^{*}, \boldsymbol{z}^{*}\right)$ is a feasible solution to $\mathbb{D}(G, \boldsymbol{w})$.

To justify this, note that $y^{\prime}((c, b))=z^{\prime}(c)=0$. Since $\phi_{b}\left(\boldsymbol{y}^{\prime}, \boldsymbol{z}^{\prime}\right) \leq w^{\prime}(b)=w(b)$, we have $-y^{\prime}((b, c))+z^{\prime}(b) \leq w(b) \leq w(a)$ by the assumption on Case 1. So $\alpha=w(a)+y^{\prime}((b, c))-z^{\prime}(b) \geq 0$ and hence $\boldsymbol{y}^{*} \geq \mathbf{0}$ and $\boldsymbol{z}^{*} \geq \mathbf{0}$. Moreover,

- $\phi_{a}\left(\boldsymbol{y}^{*}, \boldsymbol{z}^{*}\right)=-y^{*}((a, b))-y^{*}((b, a))+z^{*}(a)+z^{*}(b)=-y^{\prime}((b, c))+\alpha+z^{\prime}(b)=w(a)$;

- $\phi_{b}\left(\boldsymbol{y}^{*}, \boldsymbol{z}^{*}\right)=\phi_{b}\left(\boldsymbol{y}^{\prime}, \boldsymbol{z}^{\prime}\right)+y^{\prime}((b, c))+y^{\prime}((c, b))-y^{*}((a, b))-y^{*}((b, a))-y^{*}((c, b))+z^{*}(a)=$ $\phi_{b}\left(\boldsymbol{y}^{\prime}, \boldsymbol{z}^{\prime}\right)-y^{*}((c, b))+z^{*}(a)=\phi_{b}\left(\boldsymbol{y}^{\prime}, \boldsymbol{z}^{\prime}\right)-\alpha+\alpha \leq w^{\prime}(b)=w(b) ;$

- $\phi_{c}\left(\boldsymbol{y}^{*}, \boldsymbol{z}^{*}\right)=\phi_{c}\left(\boldsymbol{y}^{\prime}, \boldsymbol{z}^{\prime}\right)+y^{\prime}((b, c))+y^{\prime}((c, b))-z^{\prime}(b)-y^{*}((c, b)) \leq w^{\prime}(c)+y^{\prime}((b, c))-z^{\prime}(b)-\alpha=$ $w(a)+w(c)+y^{\prime}((b, c))-z^{\prime}(b)-\alpha=w(c) ;$ and

- $\phi_{v}\left(\boldsymbol{y}^{*}, \boldsymbol{z}^{*}\right)=\phi_{v}\left(\boldsymbol{y}^{\prime}, \boldsymbol{z}^{\prime}\right) \leq w^{\prime}(v)=w(v)$ for every $v \in V \backslash\{a, b, c\}$.

Combining the above observations, we conclude that $\left(\boldsymbol{y}^{*}, \boldsymbol{z}^{*}\right)$ is a feasible solution to $\mathbb{D}(G, \boldsymbol{w})$ Since $\omega^{*}$ is the optimal value of $\mathbb{D}(G, \boldsymbol{w})$, (i) and (ii) follow instantly from (1)-(3).

Case 2. $w(a)<w(b)$.

Description: Set $\alpha=w(b)-w(a)$. Let $\boldsymbol{w}^{\prime} \in \mathbb{Z}^{V^{\prime}}$ be defined by $w^{\prime}(b)=0, w^{\prime}(v)=w(v)-\alpha$ if $v \in\{c\} \cup N_{G}^{+}(c) \backslash b$, and $w^{\prime}(v)=w(v)$ if $v \in V^{\prime} \backslash\left(\{c\} \cup N_{G}^{+}(c)\right)$. Suppose $\left(\boldsymbol{y}^{\prime}, \boldsymbol{z}^{\prime}\right)$ is an integral optimal solution to $\mathbb{D}\left(G^{\prime}, \boldsymbol{w}^{\prime}\right)$. We may assume that $y^{\prime}((c, b))=0$ (otherwise, replace $y^{\prime}((c, b))$ with $y^{\prime}(c, b)+y^{\prime}(b, c)$ and $y^{\prime}((c, b))$ with 0$)$, and assume that $z^{\prime}(b)=z^{\prime}(c)=0$ (otherwise, replace $y^{\prime}((b, c))$ with $y^{\prime}((b, c))-z^{\prime}(b)-z^{\prime}(c)$, and replace both $z^{\prime}(b)$ and $z^{\prime}(c)$ with 0 . Then $\phi_{b}\left(\boldsymbol{y}^{\prime}, \boldsymbol{z}^{\prime}\right)=-y^{\prime}((b, c))+z^{\prime}(b)+z^{\prime}(c) \leq w^{\prime}(b)=0$ and $\boldsymbol{y}^{\prime} \geq \mathbf{0}, \boldsymbol{z}^{\prime} \geq \mathbf{0}$ remain valid). Define

- $y^{*}((a, b))=[-w(a)]^{+}, y^{*}((b, a))=0$, and $y^{*}(e)=y^{\prime}(e)$ if $e \in A \backslash\{(a, b),(b, a)\}$;

- $z^{*}(a)=[w(a)]^{+}, z^{*}(c)=\alpha$, and $z^{*}(v)=z^{\prime}(v)$ if $v \in V \backslash\{a, c\}$. 
Lemma 5.9. For Reduction 6 in Case 2, the following statements hold:

(i) $\left(\boldsymbol{y}^{*}, \boldsymbol{z}^{*}\right)$ is an integral optimal solution to $\mathbb{D}(G, \boldsymbol{w})$; and

(ii) the optimal value of $\mathbb{D}(G, \boldsymbol{w})$ equals that of $\mathbb{D}\left(G^{\prime}, \boldsymbol{w}^{\prime}\right)$ plus $w(b)$.

Proof. Recall the definition of $\boldsymbol{x}_{v}$ for $v \in\{a, b\}$. Since $x_{v}(v)=1$, by the complementary slackness condition we have

(1) $\phi_{v}(\boldsymbol{y}, \boldsymbol{z})=w(v)$ for each $v \in\{a, b\}$.

Let $(\boldsymbol{y}, \boldsymbol{z})$ be an optimal solution to $\mathbb{D}(G, \boldsymbol{w})$ with minimum $z(c)$. Then

(2) $y((c, b))=0$ and $z(c)=\alpha>0$.

To justify this, note that $w(b)=\phi_{b}(\boldsymbol{y}, \boldsymbol{z})=\phi_{a}(\boldsymbol{y}, \boldsymbol{z})-y((c, b))+z(c)=w(a)-y((c, b))+$ $z(c)$ by (1). Hence $-y((c, b))+z(c)=\alpha>0$, which implies $y((c, b))=0$ and $z(c)=\alpha$, for otherwise, let $(\overline{\boldsymbol{y}}, \overline{\boldsymbol{z}})$ be obtained from $(\boldsymbol{y}, \boldsymbol{z})$ by replacing $y((c, b))$ with 0 and replacing $z(c)$ with $-y((c, b))+z(c)$. Then $(\overline{\boldsymbol{y}}, \overline{\boldsymbol{z}})$ is also an optimal solution to $\mathbb{D}(G, \boldsymbol{w})$ with $\bar{z}(c)<z(c)$, contradicting the assumption on $(\boldsymbol{y}, \boldsymbol{z})$. So $(2)$ is established.

By (2) and the complementary slackness condition, $x_{a}(c)+x_{a}\left(N_{G}^{+}(c)\right)=1$. It follows from $x_{a}(b)=0$ that

(3) $x_{a}(u)=1$ and $x_{a}\left(\{c\} \cup N_{G}^{+}(c) \backslash u\right)=0$ for some vertex $u \in\{c\} \cup N_{G}^{+}(c) \backslash b$.

We propose to show that

(4) the optimal value of $\mathbb{D}\left(G^{\prime}, \boldsymbol{w}^{\prime}\right)$ is $\omega^{*}-w(b)$. So $-y^{\prime}\left(A^{\prime}\right)+z^{\prime}\left(V^{\prime}\right)=\omega^{*}-w(b)$.

To justify this, define $\left(\boldsymbol{y}^{\prime \prime}, \boldsymbol{z}^{\prime \prime}\right)$ by $y^{\prime \prime}\left((b, c)=y^{\prime \prime}((c, b))=0\right.$ and $y^{\prime \prime}(e)=y(e)$ for each $e \in A^{\prime} \backslash\{(b, c),(c, b)\}$, and $z^{\prime \prime}(b)=z^{\prime \prime}(c)=0$ and $z^{\prime \prime}(v)=z(v)$ for each $v \in V^{\prime} \backslash\{b, c\}$. Observe that

- $\phi_{b}\left(\boldsymbol{y}^{\prime \prime}, \boldsymbol{z}^{\prime \prime}\right)=-y^{\prime \prime}((b, c))-y^{\prime \prime}((c, b))+z^{\prime \prime}(b)+z^{\prime \prime}(c)=0=w^{\prime}(b)$;

- $\phi_{c}\left(\boldsymbol{y}^{\prime \prime}, \boldsymbol{z}^{\prime \prime}\right)=\phi_{c}(\boldsymbol{y}, \boldsymbol{z})+y((c, b))-z(c)-y^{\prime \prime}((b, c))-y^{\prime \prime}((c, b))+z^{\prime \prime}(c) \leq w(c)-\alpha=w^{\prime}(c)$, where the inequality follows from $(2)$;

- $\phi_{v}\left(\boldsymbol{y}^{\prime \prime}, \boldsymbol{z}^{\prime \prime}\right)=\phi_{v}(\boldsymbol{y}, \boldsymbol{z})-z(c)+z^{\prime \prime}(c) \leq w(v)-\alpha=w^{\prime}(v)$ for each $v \in N_{G}^{+}(c) \backslash b$; and

- $\phi_{v}\left(\boldsymbol{y}^{\prime \prime}, \boldsymbol{z}^{\prime \prime}\right)=\phi_{v}(\boldsymbol{y}, \boldsymbol{z}) \leq w(v)=w^{\prime}(v)$ for each $v \in V^{\prime} \backslash\left(\{c\} \cup N_{G}^{+}(c)\right)$.

Hence $\left(\boldsymbol{y}^{\prime \prime}, \boldsymbol{z}^{\prime \prime}\right)$ is a feasible solution to $\mathbb{D}\left(G^{\prime}, \boldsymbol{w}^{\prime}\right)$ with value $-y^{\prime \prime}\left(A^{\prime}\right)+z^{\prime \prime}\left(V^{\prime}\right)=-y(A)+z(V)-$ $\phi_{b}(\boldsymbol{y}, \boldsymbol{z})+\phi_{b}\left(\boldsymbol{y}^{\prime \prime}, \boldsymbol{z}^{\prime \prime}\right)=\omega^{*}-w(b)$ by $(1)$.

Note that $\left.\boldsymbol{x}_{a}\right|_{V^{\prime} \backslash b} \in K\left(G^{\prime} \backslash b\right)$. Let $x^{\prime \prime}(b)=1$ if $x_{a}(c)=0, x^{\prime \prime}(b)=0$ if $x_{a}(c)=1$, and $x^{\prime \prime}(v)=x_{a}(v)$ if $v \in V^{\prime} \backslash b$. Clearly, $\boldsymbol{x}^{\prime \prime}$ is a feasible solution to $\mathbb{P}\left(G^{\prime}, \boldsymbol{w}^{\prime}\right)$. In view of (3), we have

$$
\begin{aligned}
\left(\boldsymbol{w}^{\prime}\right)^{T} \boldsymbol{x}^{\prime \prime} & =\sum_{v \in V^{\prime} \backslash\left(\{c\} \cup N_{G}^{+}(c)\right)} w(v) x_{a}(v)+\sum_{v \in\left(\{c\} \cup N_{G}^{+}(c)\right) \backslash b}(w(v)-\alpha) x_{a}(v) \\
& =\sum_{v \in V^{\prime} \backslash\left(\{c\} \cup N_{G}^{+}(c)\right)} w(v) x_{a}(v)+(w(u)-\alpha) \\
& =\sum_{v \in V^{\prime} \backslash b} w(v) x_{a}(v)-\alpha=\sum_{v \in V^{\prime}} w(v) x_{a}(v)-\alpha=\omega^{*}-w(a)-\alpha=\omega^{*}-w(b) .
\end{aligned}
$$

Since $-y^{\prime \prime}\left(A^{\prime}\right)+z^{\prime \prime}\left(V^{\prime}\right)=\left(\boldsymbol{w}^{\prime}\right)^{T} \boldsymbol{x}^{\prime \prime}=\omega^{*}-w(b)$, we obtain (4) using the LP-duality theorem.

(5) $\left(\boldsymbol{y}^{*}, \boldsymbol{z}^{*}\right)$ is a feasible solution to $\mathbb{D}(G, \boldsymbol{w})$.

To justify this, observe that

- $\phi_{a}\left(\boldsymbol{y}^{*}, \boldsymbol{z}^{*}\right)=-y^{*}((a, b))-y^{*}((b, a))+z^{*}(a)+z^{*}(b)=-[-w(a)]^{+}+[w(a)]^{+}+z^{\prime}(b)=w(a)$;

- $\phi_{b}\left(\boldsymbol{y}^{*}, \boldsymbol{z}^{*}\right)=\phi_{a}\left(\boldsymbol{y}^{*}, \boldsymbol{z}^{*}\right)-y^{*}((c, b))+z^{*}(c) \leq w(a)+\alpha=w(b)$; 
- $\phi_{c}\left(\boldsymbol{y}^{*}, \boldsymbol{z}^{*}\right)=\phi_{c}\left(\boldsymbol{y}^{\prime}, \boldsymbol{z}^{\prime}\right)+y^{\prime}((b, c))-z^{\prime}(c)+z^{*}(c) \leq w^{\prime}(c)+\alpha=w(c)$;

- $\phi_{v}\left(\boldsymbol{y}^{*}, \boldsymbol{z}^{*}\right)=\phi_{v}\left(\boldsymbol{y}^{\prime}, \boldsymbol{z}^{\prime}\right)-z^{\prime}(c)+z^{*}(c) \leq w^{\prime}(v)+\alpha=w(v)$ for each $v \in N_{G}^{+}(c) \backslash b$; and

- $\phi_{v}\left(\boldsymbol{y}^{*}, \boldsymbol{z}^{*}\right)=\phi_{v}\left(\boldsymbol{y}^{\prime}, \boldsymbol{z}^{\prime}\right) \leq w^{\prime}(v)=w(v)$ for each $v \in V^{\prime} \backslash\left(\{c\} \cup N_{G}^{+}(c)\right)$.

Therefore (5) holds.

(6) $-y^{*}(A)+z^{*}(V)=\omega^{*}$.

Indeed, since $z^{\prime}(c)=0$ and $y^{\prime}((b, c))=0$, we obtain $-y^{\prime}\left(A^{\prime} \backslash(b, c)\right)+z^{\prime}\left(V^{\prime} \backslash c\right)=\omega^{*}-w(b)$ by (4). It follows that $-y^{*}(A)+z^{*}(V)=-y^{\prime}\left(A^{\prime} \backslash(b, c)\right)-y^{*}((a, b))-y^{*}((b, a))+z^{\prime}\left(V^{\prime} \backslash c\right)+z^{*}(a)+$ $z^{*}(c)=\omega^{*}-w(b)-[-w(a)]^{+}+[w(a)]^{+}+\alpha=\omega^{*}-w(b)+w(a)+\alpha=\omega^{*}$, as desired.

Since $\omega^{*}$ is the optimal value of $\mathbb{D}(G, \boldsymbol{w})$, (i) and (ii) follow instantly from (4)-(6).

\section{$6 \quad$ Integralities}

Given the structural description and reduction operations presented in the previous sections, we are ready to establish the main result now.

Proof of Theorem 1.5. Implication $(i i i) \Rightarrow(i i)$ follows directly from the Edmonds-Giles theorem [5] stated in Section 1. Implication $(i i) \Rightarrow(i)$ is given by Lemma 3.1 and Theorem 3.2. It remains to show implication $(i) \Rightarrow($ iii).

Let $G=(V, A)$ be a permissible digraph. To prove that $G$ is kernel Mengerian; that is, $\mathbb{D}(G, \boldsymbol{w})$ has an integral optimal solution for any $\boldsymbol{w} \in \mathbb{Z}^{V}$, we apply induction on $|V|+|A|$. The statement holds trivially when $|V|+|A| \leq 4$. So we proceed to the induction step, and assume that every permissible digraph $G^{\prime}=\left(V^{\prime}, A^{\prime}\right)$, with $\left|V^{\prime}\right|+\left|A^{\prime}\right|<|V|+|A|$, is kernel Mengerian. By induction hypothesis, we may further assume that

- the underlying graph of $G$ is connected; and

- none of Reductions 1-6 in Section 5 is applicable to $G$ (see Lemmas 5.1, 5.2, 5.4, and 5.5).

Throughout this section we reserve symbol $H$ for a strongly connected component of $G$ with no outgoing arcs (that is, $G$ contains no arc from $V(H)$ to $V \backslash V(H)$ ).

Claim 6.1. The following statements hold for $H$ :

(i) $H$ contains at least two vertices;

(ii) $H$ is bipartite; and

(iii) no two vertices in $H$ have a common in-neighbor outside $H$.

Since Reduction 1 does not apply to $G$, we have (i). Statement (ii) follows directly from (1.1). To prove statement (iii), suppose on the contrary that some vertices $a$ and $b$ in $H$ have a common in-neighbor $c$ outside $H$. Let $Q_{a}$ (resp. $Q_{b}$ ) be a directed path from $a$ to $b$ (resp. from $b$ to $a$ ) in $H$. Then $Q_{a} \cup Q_{b} \cup\{(c, a),(c, b)\}$ would contain a gear, a contradiction. Thus Claim 6.1 is established.

Claim 6.2. $H$ is obtained from an induced even cycle $C=v_{1} v_{2} \ldots v_{2 t} v_{1}$, with $t \geq 3$, by replacing each even-indexed vertex $v_{2 i}$, for $1 \leq i \leq t$, with a stable set $S_{2 i}$ of size at least two, with $v_{2 i} \in S_{2 i}$, such that

- there is an arc from $v_{2 i-1}$ to each vertex in $S_{2 i}$;

- there is an arc from each vertex in $S_{2 i}$ to $v_{2 i+1}$; and 
- $\left\{v_{1}, v_{3}, \ldots, v_{2 t-1}\right\}, S_{2}, S_{4}, \ldots, S_{2 t}$ are pairwise disjoint, where $v_{2 t+1}=v_{1}$. (So $H$ has $2 \sum_{i=1}^{t}\left|S_{2 i}\right|$ arcs in total.)

To justify this, recall that $H$ has a tree-like structure defined by a tree $T$ rooted at a vertex $r$; see Theorem 4.1 for its structural description and undefined notations involved in our proof. Observe that

(1) $T$ has at least two vertices.

Otherwise, $H$ is an induced cycle by Theorem 4.1. Since $H$ has no outgoing arcs, it cannot contain four or more vertices, for otherwise Reduction 2 applies to three consecutive vertices on $H$ (in view of Claim 6.1(iii)). Since $G$ contains no odd cycle, $H$ has only two vertices $a$ and $b$, where $b$ has a neighbor $c$ outside $H$. Now $N_{G}^{+}(a)=\{b\}, N_{G}^{+}(b)=\{a\}$, and $(c, b) \in A$ is a cut arc of $G$ by Theorem 4.4. So either Reduction 5 or Reduction 6 applies to $\{a, b, c\}$; this contradiction proves (1).

For each vertex $v$ in $T$, let $R_{v}$ be the path from $r$ to $v$; we call $\left|R_{v}\right|$ the level of $v$ in $T$. Let us consider an arbitrary vertex pair $(v, x)$ in $T$, such that

(2) $x(\neq r)$ is a leaf of $T$ and $v$ is its parent;

(3) $x$ has the highest level among all leaves of $T$; and

(4) each internal vertex (if any) of $P_{v, x}$, the subpath of $P_{v}$ between $s_{x}$ and $t_{x}$, has degree two in $H$.

The existence of such a pair is guaranteed by (1) and Theorem 4.1(vi).

(5) Each internal vertex of $P_{x}$ (if any) has degree two in $H$. Moreover, $\left|P_{x}\right| \leq 3$, with equality only when $\left(s_{x}, t_{x}\right) \in A$ and $\left|N_{H}^{+}\left(t_{x}\right)\right| \geq 2$.

The first half of this statement follows instantly from Theorem 4.1(ii) and (iii). Let $a b t_{x}$ be a subpath of $P_{x}$ if $\left|P_{x}\right| \geq 3$, and let $c$ be an out-neighbor of $t_{x}$. Note that $s_{x} \neq t_{x}$ if $\left|P_{x}\right|=3$ because $H$ contains no odd cycle. Since Reduction 2 does not apply to $\left\{a, b, t_{x}\right\}$ or to $\left\{b, t_{x}, c\right\}$ (see Claim 6.1(iii)), we get the second half of (5).

(6) If $\left|P_{x}\right|=3$, then $v \neq r$.

Assume on the contrary that $v=r$. By (5), we have $\left(s_{x}, t_{x}\right) \in A$ and $\left|N_{H}^{+}\left(t_{x}\right)\right| \geq 2$. As $P_{r}$ is an induced cycle, it contains $\left(s_{x}, t_{x}\right)$. Let $u$ be an out-neighbor of $t_{x}$ outside $P_{r} \cup P_{x}$. From Theorem 4.1(ii) and (iii), we see that $\left(t_{x}, u\right)$ is contained in $P_{y}$ for some child $y$ other than $x$ of $r$ in $T$. Since $s_{y}=t_{x}$ and $\left|P_{r}\left[s_{x}, s_{y}\right]\right|$ is odd, $P_{r} \cup P_{x} \cup P_{y}$ is a ring; this contradiction justifies (6).

(7) If $P_{v, x}=P_{v}\left[t_{x}, s_{x}\right]$, then $\left|P_{v, x}\right|=0$ or $v=r$ or $\left|N_{H}^{+}\left(t_{x}\right)\right|=1$.

Assume the contrary: $\left|P_{v, x}\right| \geq 1, v \neq r$, and $t_{x}$ has an out-neighbor $u \notin P_{v, x}$. By Theorem 4.1(v), we have $t_{x} \neq s_{v}$. By Theorem 4.1(ii) and (iii), arc $\left(t_{x}, u\right)$ is contained in $P_{y}$ for some child $y$ of $v$ other than $x$ in $T$. Since $s_{y}=t_{x}$, by Theorem 4.1(viii), we obtain $t_{y} \in P_{v}\left(s_{y}, t_{v}\right]$, which together with Theorem 4.1(iv) implies $s_{y}=s_{v}$, a contradiction. So (7) holds.

(8) $s_{y} \neq t_{y}$ for every child $y$ of $v$ in $T$.

Suppose on the contrary that $P_{y}$ is a cycle. Since $y$ is a leaf of $T$ with the highest level, we have $\left|P_{y}\right|=2$ by (5) (with $(v, y)$ in place of $(v, x)$ ). Let $a$ and $b$ be the two vertices in $P_{y}$, where $b=s_{y}$, and let $c$ be an out-neighbor of $b$ on $P_{v}$. Then Reduction 4 applies to $\{a, b, c\}$; this contradiction establishes (8). 
(9) We may assume that $\left|P_{x}\right| \geq 2$.

By (8), we have $\left|P_{v, x}\right| \geq 1$. Let us first consider the case when $P_{v, x}=P_{v}\left[s_{x}, t_{x}\right]$. Since $G$ contains no parallel arcs, $\left|P_{x}\right|+\left|P_{v, x}\right| \geq 3$. By (4) and (5), each internal vertex of $P_{v, x}$ and that of $P_{x}$ has degree two in $H$. Swapping $P_{v, x}$ and $P_{x}$ if necessary, we may assume that $\left|P_{x}\right| \geq 2$.

It remains to consider the case when $P_{v, x}=P_{v}\left[t_{x}, s_{x}\right]$. Assume the contrary: $\left|P_{x}\right|=1$. Thus $\left(s_{x}, t_{x}\right) \in A$. Since $H$ contains no odd cycle, $\left|P_{v, x}\right|$ is odd. As Reduction 2 does not apply to any three consecutive vertices on $P_{v}\left(t_{x}, s_{x}\right]$ (see (4)), we have $\left|P_{v, x}\right| \leq 3$, with equality only when $\left(t_{x}, s_{x}\right) \in A$. Thus $H$ contains both $\left(s_{x}, t_{x}\right)$ and $\left(t_{x}, s_{x}\right)$ no matter whether $\left|P_{v, x}\right|=3$ or 1 .

By Theorem 4.1(v), we have $t_{x} \neq s_{v}$. Let $D$ be a cycle containing $P_{v}$ in $\cup_{z \in V\left(R_{v}\right)} P_{z}$ (see (1) in the proof of Theorem 4.1), and let $c$ be the out-neighbor of $s_{x}$ on $D$. Clearly, $c \neq t_{x}$. If $\left|P_{v, x}\right|=3$, then $D \cup\left\{\left(s_{x}, t_{x}\right),\left(t_{x}, s_{x}\right)\right\}$ would be a ring. So we assume $\left|P_{v, x}\right|=1$. Since Reduction 4 does not apply to $\left\{t_{x}, s_{x}, c\right\}$, we have $\left|N_{H}^{+}\left(t_{x}\right)\right| \geq 2$ and hence $v=r$ by (7). Thus $P_{r}$ is not an induced cycle as one of $\left(s_{x}, t_{x}\right)$ and $\left(t_{x}, s_{x}\right)$ is outside $P_{r}$, contradicting Theorem 4.1(i). So (9) holds.

(10) $\left|P_{x}\right|=2$.

Otherwise, from (5), (6), and (9) we see that $\left|P_{x}\right|=3, v \neq r,\left(s_{x}, t_{x}\right) \in A$, and $\left|N_{H}^{+}\left(t_{x}\right)\right| \geq 2$. By (7), we have $t_{x} \in P_{v}\left(s_{x}, t_{v}\right]$. Thus Theorem 4.1(iv) enforces $s_{x}=s_{v}$. Let $u$ be an out-neighbor of $t_{x}$ such that arc $\left(t_{x}, u\right)$ is outside $P_{v}$. In view of Theorem 4.1(ii) and (iii), $t_{x}$ is an internal vertex of $P_{v}$ and $\left(t_{x}, u\right)$ belongs to $P_{y}$ for some child $y$ of $v$ in $T$. Thus the existence of both $P_{x}$ and $P_{y}$ contradicts Theorem 4.1(vii), and hence (10) is justified.

(11) $\left|P_{v, x}\right|=2$ and $P_{v, x}=P_{v}\left[s_{x}, t_{x}\right]$. Moreover, if $v \neq r$, then $s_{x}=s_{v}$ and $t_{x}$ is an internal vertex of $P_{v}$.

By (8), we have $\left|P_{v, x}\right| \geq 1$. By Claim 6.1(ii), $\left|P_{v, x}\right|$ has the same parity as $\left|P_{x}\right|$ and hence is even by (10). Since Reduction 2 does not apply to any three consecutive vertices on $P_{v, x}$ (see Claim 6.1(iii) and (4)), we have $\left|P_{v, x}\right|=2$. As Reduction 3 does not apply to the four vertices in $P_{x} \cup P_{v, x}$, we further obtain $P_{v, x}=P_{v}\left[s_{x}, t_{x}\right]$. If $v \neq r$, then $s_{x}=s_{v}$ by Theorem 4.1(iv) and hence $t_{x}$ is an internal vertex of $P_{v}$ by Theorem 4.1(ii). Thus (11) follows.

(12) For each in-neighbor $u$ of $t_{x}$ outside $P_{v}$, we have $N_{H}^{-}(u)=\left\{s_{x}\right\}$ and $N_{H}^{+}(u)=\left\{t_{x}\right\}$.

By (11) and Theorem 4.1(ii), arc $\left(u, t_{x}\right)$ is contained in $P_{y}$ for some child $y$ of $v$ in $T$. If $v \neq r$, then $s_{y}=s_{x}=s_{v}$ by (11) and Theorem 4.1(iv) and (vii). If $v=r$, we also have $s_{y}=s_{x}$ by Theorem 4.1(ix). Since $y$ has the same level as $x$ in $T$, by (5), (10), and (11) (with $(v, y)$ in place of $(v, x))$, we have $N_{H}^{-}(u)=\left\{s_{x}\right\}$ and $N_{H}^{+}(u)=\left\{t_{x}\right\}$. This proves (12).

(13) $\left|N_{H}^{+}\left(t_{x}\right)\right| \geq 2$.

Suppose one the contrary that $t_{x}$ has only one out-neighbor, say $a$. Then $a \in P_{v}$ by (11). If $N_{H}^{+}(a)=\{b\}$ and $b \neq s_{x}$, then Reduction 2 applies to $\left\{t_{x}, a, b\right\}$ by (12), a contradiction. If $N_{H}^{+}(a)=\left\{s_{x}\right\}$, then Reduction 3 applies to the four vertices on $P_{v}\left[s_{x}, a\right]$, again a contradiction. So $\left|N_{H}^{+}(a)\right| \geq 2$. Let $R_{v}$ be the path from $r$ to $v$ in $T$ and let $D$ be a shortest cycle in $\cup_{q \in V\left(R_{v}\right)} P_{q}$ containing $P_{v}$ (see (1) in the proof of Theorem 4.1). Then $D$ contains precisely one out-neighbor of $a$, say $b$. Let $c$ be an out-neighbor of $a$ outside $D$. Since $H$ is strongly connected, it contains a path $Q$ from $a$ to a vertex on $D$, passing through $(a, c)$, such that all internal vertices of $Q$ are outside $D$. Thus $P_{v}\left[s_{x}, a\right] \cup P_{x} \cup Q$ would be gear if $Q$ is a cycle and $D \cup P_{x} \cup Q$ would be a ring otherwise; this contradiction establishes (13). 
(14) $v=r$ and hence $T$ is a star centered at $v$.

Suppose on the contrary that $v \neq r$. By (11), $s_{x}=s_{v}$ and $t_{x}$ is an internal vertex of $P_{v}$. By (13), $t_{x}$ has an out-neighbor $u$ such that arc $\left(t_{x}, u\right)$ is outside $P_{v}$. By Theorem 4.1(ii), arc $\left(t_{x}, u\right)$ is contained in $P_{y}$ for some child $y$ of $v$ in $T$, which contradicts Theorem 4.1(vii). So (14) is true.

(15) Let $y$ be a child of $r$ in $T$ such that $s_{y}=t_{x}$ and, subject to this, $\left|P_{r}\left[s_{y}, t_{y}\right]\right|$ is as small as possible. Then each internal vertex (if any) of $P_{r}\left[s_{y}, t_{y}\right]$ has degree two in $H$.

Suppose on the contrary that some internal vertex of $P_{r}\left[s_{y}, t_{y}\right]$ has degree at least three in $H$. By (14), $r$ has a child $z$ in $T$ such that at least one end of $P_{z}$ is on $P_{r}\left(s_{y}, t_{y}\right)$. By Theorem 4.1(vi), both $s_{z}$ and $t_{z}$ are on $P_{r}\left[s_{y}, t_{y}\right]$. By (8), Theorem 4.1(ix), and (11) (with $y$ in place of $x$ ), we obtain $t_{z} \in P_{r}\left(s_{z}, t_{y}\right]$. By Theorem 4.1(ix), we further have $s_{z}=s_{y}$. Thus $\left|P_{r}\left[s_{z}, t_{z}\right]\right|<\left|P_{r}\left[s_{y}, t_{y}\right]\right|$, contradicting the hypothesis on $y$. Therefore (15) holds.

By (13), the vertex $y$ specified in (15) is available. From (5), (10), and (11) (with $y$ in place of $x)$, we see that $\left|P_{y}\right|=\left|P_{r}\left[s_{y}, t_{y}\right]\right|=2$ and that both the internal vertex of $P_{y}$ and that of $P_{r}\left[s_{y}, t_{y}\right]$ have degree two. The process can be repeated by replacing $x$ with $y$. Let $X$ be the set of all children $x$ of $r$ in $T$, such that each internal vertex of $P_{r}\left[s_{x}, t_{x}\right]$ has degree two in $H$ (see (4) and (11)). From the above observation, we deduce that $P_{r} \cup\left(\cup_{x \in X} P_{x}\right)$ is obtained from $P_{r}=v_{1} v_{2} \ldots v_{2 t} v_{1}$ by replacing each even-indexed vertex $v_{2 i}$ with a stable set $S_{2 i}$ of size at least two such that

- there is an arc from $v_{2 i-1}$ to each vertex in $S_{2 i}$;

- there is an arc from each vertex in $S_{2 i}$ to $v_{2 i+1}$; and

- $\left\{v_{1}, v_{3}, \ldots, v_{2 t-1}\right\}, S_{2}, S_{4}, \ldots, S_{2 t}$ are pairwise disjoint,

where $v_{2 t+1}=v_{1}$. For convenience, we view $v_{2 i}$ as a vertex in $S_{2 i}$ for $1 \leq i \leq t$. Observe that $t \geq 3$, for otherwise Reduction 3 would apply to the four vertices on $P_{r}$.

Let $y$ be an arbitrary child of $r$ in $T$. Since the terminus $t_{y}$ of $P_{y}$ is some $v_{2 i-1}$, by (12) we have $y \in X$, and hence $H=P_{r} \cup\left(\cup_{x \in X} P_{x}\right)$. Therefore Claim 6.2 is established.

We shall rely heavily on the structural description of $H$ given in Claim 6.2. Let $B=$ $\left\{\left(v_{1}, u\right): u \in S_{2} \backslash v_{2}\right\}$, let $G^{\prime}=\left(V, A^{\prime}\right)$ be the digraph $G \backslash B$, and let $H^{\prime}=H \backslash B$. Set $K_{1}=$ $\left\{v_{1}, v_{3}, \ldots, v_{2 t-1}\right\}$ and $K_{2}=V(H) \backslash K_{1}$. Observe that

(16) $K_{1}$ and $K_{2}$ are the only two kernels in each of $H$ and $H^{\prime}$. Thus the restriction of any kernel of $G$ to $H$ is either $K_{1}$ or $K_{2}$.

In the remainder of our proof, we reserve the symbol $\boldsymbol{x}^{\prime}$ for an integral optimal solution to $\mathbb{P}\left(G^{\prime}, \boldsymbol{w}\right)$ and the pair $\left(\boldsymbol{y}^{\prime}, \boldsymbol{z}^{\prime}\right)$ for an integral optimal solution to $\mathbb{D}\left(G^{\prime}, \boldsymbol{w}\right)$ such that

(17) $z^{\prime}\left(v_{1}\right)$ is as small as possible.

It follows instantly from (16) that

(18) $\boldsymbol{x}^{\prime}$ is also a feasible solution to $\mathbb{P}(G, \boldsymbol{w})$.

Claim 6.3. $H=G$.

Assume the contrary: $H \neq G$. Let us define $\overline{\boldsymbol{w}} \in \mathbb{Z}^{V(H)}$ as

- $\bar{w}(v)=w(v)+\sum_{u \in N_{G}^{-}(v) \backslash V(H)} y^{\prime}((u, v))-z^{\prime}\left(N_{G}^{-}(v) \backslash V(H)\right)$ for each $v \in V(H)$. 
Set $\overline{\boldsymbol{x}}=\left.\boldsymbol{x}^{\prime}\right|_{V(H)}$. Using optimality of $\boldsymbol{x}^{\prime}$ and $\left(\boldsymbol{y}^{\prime}, \boldsymbol{z}^{\prime}\right)$, it is easy to check that $\overline{\boldsymbol{x}}$ and $\left(\left.\boldsymbol{y}^{\prime}\right|_{A\left(H^{\prime}\right)},\left.\boldsymbol{z}^{\prime}\right|_{V(H)}\right)$ are feasible solutions to $\mathbb{P}\left(H^{\prime}, \overline{\boldsymbol{w}}\right)$ and $\mathbb{D}\left(H^{\prime}, \overline{\boldsymbol{w}}\right)$, respectively, and satisfy the complementary slackness condition (because $H$ has no outgoing arcs). So

(19) $\overline{\boldsymbol{x}}$ is an integral optimal solution to $\mathbb{P}\left(H^{\prime}, \overline{\boldsymbol{w}}\right)$ and hence also an integral optimal solution to $\mathbb{P}(H, \overline{\boldsymbol{w}})$ by (16). Furthermore, $\overline{\boldsymbol{w}}^{T} \overline{\boldsymbol{x}}=-y^{\prime}\left(A\left(H^{\prime}\right)\right)+z^{\prime}(V(H))$.

Let $(\overline{\boldsymbol{y}}, \overline{\boldsymbol{z}})$ be an integral optimal solution to $\mathbb{D}(H, \overline{\boldsymbol{w}})$. By $(19)$, we have

(20) $\overline{\boldsymbol{w}}^{T} \overline{\boldsymbol{x}}=-\bar{y}(A(H))+\bar{z}(V(H))$.

Now let us define $\boldsymbol{y}^{*} \in \mathbb{Z}^{A}$ and $\boldsymbol{z}^{*} \in \mathbb{Z}^{V}$ as

- $\left.\boldsymbol{y}^{*}\right|_{A(H)}=\overline{\boldsymbol{y}}$ and $\left.\boldsymbol{y}^{*}\right|_{A \backslash A(H)}=\left.\boldsymbol{y}^{\prime}\right|_{A \backslash A(H)}$;

- $\left.\boldsymbol{z}^{*}\right|_{V(H)}=\overline{\boldsymbol{z}}$ and $\left.\boldsymbol{z}^{*}\right|_{V \backslash V(H)}=\left.\boldsymbol{z}^{\prime}\right|_{V \backslash V(H)}$.

Since $H$ has no outgoing arcs, the definition of $\overline{\boldsymbol{w}}$ guarantees that $\left(\boldsymbol{y}^{*}, \boldsymbol{z}^{*}\right)$ is a feasible solution to $\mathbb{D}(G, \boldsymbol{w})$. Moreover,

$$
\begin{aligned}
-y^{*}(A)+z^{*}(V) & =-\bar{y}(A(H))+\bar{z}(V(H))-y^{\prime}(A \backslash A(H))+z^{\prime}(V \backslash V(H)) \\
& =\overline{\boldsymbol{w}}^{T} \overline{\boldsymbol{x}}-y^{\prime}(A \backslash A(H))+z^{\prime}(V \backslash V(H)) \\
& =\overline{\boldsymbol{w}}^{T} \overline{\boldsymbol{x}}+y^{\prime}\left(A\left(H^{\prime}\right)\right)-z^{\prime}(V(H))-y^{\prime}\left(A^{\prime}\right)+z^{\prime}(V) \\
& =-y^{\prime}\left(A^{\prime}\right)+z^{\prime}(V) \\
& =\boldsymbol{w}^{T} \boldsymbol{x}^{\prime}
\end{aligned}
$$

where the second equality follows from (20) and the fourth one from (19). Thus, by (18) and the LP-duality theorem, $\boldsymbol{x}^{\prime}$ and $\left(\boldsymbol{y}^{*}, \boldsymbol{z}^{*}\right)$ are integral optimal solution to $\mathbb{P}(G, \boldsymbol{w})$ and $\mathbb{D}(G, \boldsymbol{w})$, respectively. Therefore we may assume that $H=G$, otherwise the desired statement of Theorem 1.5 has been established.

Claim 6.4. $z^{\prime}\left(v_{1}\right) \geq 1$.

Otherwise, $z^{\prime}\left(v_{1}\right)=0$. Let us define $\boldsymbol{y}^{*} \in \mathbb{Z}^{A}$ by

- $\left.\boldsymbol{y}^{*}\right|_{A^{\prime}}=\left.\boldsymbol{y}^{\prime}\right|_{A^{\prime}}$ and $y^{*}\left(\left(v_{1}, u\right)\right)=0$ for each $\left(v_{1}, u\right) \in B$.

Then $\left(\boldsymbol{y}^{*}, \boldsymbol{z}^{\prime}\right)$ is a feasible solution to $\mathbb{D}(G, \boldsymbol{w})$ with value $\boldsymbol{w}^{T} \boldsymbol{x}^{\prime}$. Thus, by (18) and the LP-duality theorem, $\boldsymbol{x}^{\prime}$ and $\left(\boldsymbol{y}^{*}, \boldsymbol{z}^{\prime}\right)$ are integral optimal solution to $\mathbb{P}(G, \boldsymbol{w})$ and $\mathbb{D}(G, \boldsymbol{w})$, respectively. So we may assume that $z^{\prime}\left(v_{1}\right) \geq 1$.

Claim 6.5. $\phi_{u}\left(\boldsymbol{y}^{\prime}, \boldsymbol{z}^{\prime}\right)=w(u)$ for all $u \in K_{2}$.

Assume the contrary: $\phi_{u}\left(\boldsymbol{y}^{\prime}, \boldsymbol{z}^{\prime}\right)<w(u)$ for some $u \in K_{2}$, say $u \in S_{2 i}$ for some $i$ with $1 \leq i \leq t$ (recall Claim 6.2). Set $\epsilon=\min \left\{w(u)-\phi_{u}\left(\boldsymbol{y}^{\prime}, \boldsymbol{z}^{\prime}\right), z^{\prime}\left(v_{1}\right)\right\}$. From Claim 6.4, we see that $\epsilon$ is a positive integer. Let us define $\boldsymbol{y} \in \mathbb{Z}^{A^{\prime}}$ and $\boldsymbol{z} \in \mathbb{Z}^{V}$ as

- $y(e)=y^{\prime}(e)+\epsilon$ if $e=\left(v_{2 j-1}, v_{2 j}\right)$ for $i+1 \leq j \leq t$, and $y(e)=y^{\prime}(e)$ otherwise;

- $z\left(v_{1}\right)=z^{\prime}\left(v_{1}\right)-\epsilon, z\left(v_{2 j}\right)=z^{\prime}\left(v_{2 j}\right)+\epsilon$ for $i+1 \leq j \leq t, z(u)=z^{\prime}(u)+\epsilon$, and $z(v)=z^{\prime}(v)$ otherwise.

Using Claims 6.2 and 6.3 , it is a routine matter to check that $(\boldsymbol{y}, \boldsymbol{z})$ is a feasible solution to $\mathbb{D}\left(G^{\prime}, \boldsymbol{w}\right)$. Since $-y\left(A^{\prime}\right)+z(V)=-\left(y^{\prime}\left(A^{\prime}\right)+(t-i) \epsilon\right)+z^{\prime}(V)-\epsilon+(t-i+1) \epsilon=-y^{\prime}\left(A^{\prime}\right)+z^{\prime}(V)$, pair $(\boldsymbol{y}, \boldsymbol{z})$ is also an integral optimal solution to $\mathbb{D}\left(G^{\prime}, \boldsymbol{w}\right)$. As $z\left(v_{1}\right)<z^{\prime}\left(v_{1}\right)$, the existence of $(\boldsymbol{y}, \boldsymbol{z})$ contradicts the choice of $\left(\boldsymbol{y}^{\prime}, \boldsymbol{z}^{\prime}\right)$ (see (17)). Thus Claim 6.5 is justified.

Claim 6.6. $z^{\prime}(u)=0$ for all $u \in K_{1} \backslash v_{1}$. 
Assume the contrary: $z^{\prime}\left(v_{2 i+1}\right)>0$ for some $i$ with $1 \leq i \leq t-1$. Set $\epsilon=\min \left\{z^{\prime}\left(v_{2 i+1}\right), z^{\prime}\left(v_{1}\right)\right\}$. By Claim 6.4, $\epsilon$ is a positive integer. Let us define $\boldsymbol{y} \in \mathbb{Z}^{A^{\prime}}$ and $\boldsymbol{z} \in \mathbb{Z}^{V}$ as

- $y(e)=y^{\prime}(e)+\epsilon$ if $e=\left(v_{2 j+1}, v_{2 j+2}\right)$ for $1 \leq j \leq t-1$ and $j \neq i$, and $y(e)=y^{\prime}(e)$ otherwise;

- $z\left(v_{1}\right)=z^{\prime}\left(v_{1}\right)-\epsilon, z\left(v_{2 i+1}\right)=z^{\prime}\left(v_{2 i+1}\right)-\epsilon, z\left(v_{2 j}\right)=z^{\prime}\left(v_{2 j}\right)+\epsilon$ for $1 \leq j \leq t$, and $z(v)=z^{\prime}(v)$ otherwise.

From Claims 6.2 and 6.3 , it is easy to see that $(\boldsymbol{y}, \boldsymbol{z})$ is a feasible solution to $\mathbb{D}\left(G^{\prime}, \boldsymbol{w}\right)$. Since $-y\left(A^{\prime}\right)+z(V)=-\left(y^{\prime}\left(A^{\prime}\right)+(t-2) \epsilon\right)+z^{\prime}(V)-2 \epsilon+t \epsilon=-y^{\prime}\left(A^{\prime}\right)+z^{\prime}(V)$, pair $(\boldsymbol{y}, \boldsymbol{z})$ is also an integral optimal solution to $\mathbb{D}\left(G^{\prime}, \boldsymbol{w}\right)$. As $z\left(v_{1}\right)<z^{\prime}\left(v_{1}\right)$, the existence of $(\boldsymbol{y}, \boldsymbol{z})$ contradicts the choice of $\left(\boldsymbol{y}^{\prime}, \boldsymbol{z}^{\prime}\right)$ (see (17)). This proves Claim 6.6.

Claim 6.7. Let $\boldsymbol{x}^{*} \in \mathbb{Z}^{V}$ be defined by $x^{*}(u)=1$ if $u \in K_{2}$ and 0 otherwise. Then $\boldsymbol{x}^{*}$ is an integral optimal solution to $\mathbb{P}(G, \boldsymbol{w})$.

To justify this, note first that $G=H$ by Claim 6.3. So, using (16), $\boldsymbol{x}^{*}$ is a feasible solution to $\mathbb{P}(G, \boldsymbol{w})$. By Claim 6.5, we have $w\left(K_{2}\right)=\sum_{u \in K_{2}} \phi_{u}\left(\boldsymbol{y}^{\prime}, \boldsymbol{z}^{\prime}\right)=-y^{\prime}\left(A^{\prime}\right)+z^{\prime}(V)$, where the second equality holds because $z^{\prime}(u)=0$ for all $u \in K_{1} \backslash v_{1}$ by Claim 6.6, and $z^{\prime}\left(v_{1}\right)$ appears in none of $\phi_{u}\left(\boldsymbol{y}^{\prime}, \boldsymbol{z}^{\prime}\right)$ with $u \neq v_{2}$ (recall that $\left.G^{\prime}=G \backslash B\right)$. From the LP-duality theorem, we thus conclude that $\boldsymbol{x}^{*}$ is an integral optimal solution to $\mathbb{P}\left(G^{\prime}, \boldsymbol{w}\right)$, and hence also an integral optimal solution to $\mathbb{P}(G, \boldsymbol{w})$ by $(16)$.

Let $\left(\boldsymbol{y}^{*}, \boldsymbol{z}^{*}\right)$ be an optimal solution to $\mathbb{D}(G, \boldsymbol{w})$. Observe that

(21) $z^{*}(u)=0$ for all $u \in K_{1}$.

Indeed, by definition, $x^{*}(u)=1$ for each $u \in K_{2}$. So $x^{*}(u)+x^{*}\left(N_{G}^{+}(u)\right) \geq 2$ for each $u \in K_{1}$. Thus (21) follows instantly from the optimality established in Claim 6.7 and the complementary slackness condition.

Claim 6.8. $\mathbb{D}(G, \boldsymbol{w})$ has an integral optimal solution.

To justify this, let $\mathbb{D}^{\prime}$ denote the linear program obtained from $\mathbb{D}(G, \boldsymbol{w})$ by deleting the variables $z(u)$ for all $u \in K_{1}$. It follows from (21) that the optimal value of $\mathbb{D}^{\prime}$ is at least $y^{*}(A)+z^{*}(V)$. By Claims 6.2 and $6.3, G$ is bipartite. So the incidence matrix $M_{1}$ of the underlying graph of $G$ is totally unimodular. Let $M_{2}$ be the matrix formed by the columns of $M_{1}$ corresponding to arcs leaving $K_{2}$. Observe that for each $u \in K_{2}$ and the unique arc $e$ leaving $u$, the variables $z(u)$ and $y(e)$ appear, with opposite coefficients, in exactly the same inequalities of $\mathbb{D}^{\prime}$. Clearly, the constraint matrix of $\mathbb{D}^{\prime}$ is $\left(-M_{2}, M_{2}\right)$, which is totally unimodular. Hence $\mathbb{D}^{\prime}$ has an integral optimal solution $\left(\boldsymbol{y}^{\prime}, \boldsymbol{z}^{\prime}\right)$ with value $y^{\prime}(A)+z^{\prime}\left(K_{2}\right) \geq y^{*}(A)+z^{*}(V)$. Let $\boldsymbol{y} \in \mathbb{Z}^{A}$ and $\boldsymbol{z} \in \mathbb{Z}^{V}$ be defined by $\boldsymbol{y}=\boldsymbol{y}^{\prime}, z(u)=z^{\prime}(u)$ if $u \in K_{2}$, and $z(u)=0$ if $u \in K_{1}$. Then $(\boldsymbol{y}, \boldsymbol{z})$ is an integral solution to $\mathbb{D}(G, \boldsymbol{w})$ with value $y(A)+z(V) \geq y^{*}(A)+z^{*}(V)$. Therefore it is also optimal.

This completes the proof of Claim 6.8 and hence of Theorem 1.5.

Acknowledgment. We are indebted to an anonymous referee for discovering an error in our original proof and a shorter proof of Claim 6.8. We greatly appreciate his/her insightful skepticism and invaluable comments and suggestions. 


\section{References}

[1] R. Aharoni and R. Holzman, Fractional kernels in digraphs, J. Combin. Theory Ser. B 73 (1998), 1-6.

[2] J. Bang-Jensen and G. Gutin, Digraphs: Theory, Algorithms and Applications, Springer, 2001.

[3] J.A. Bondy and U.S.R. Murty, Graph Theory, Springer, 2008.

[4] E. Boros and V. Gurvich, Perfect graphs are kernel-solvable, Discrete Math. 159 (1996), $35-55$.

[5] J. Edmonds and R. Giles, A min-max relation for submodular functions on graphs, Ann. Discrete Math. 1 (1977), 185-204.

[6] Egres Open (Categories: Stable Matchings and Kernels and Integer Polyhedra), Egerváry Research Group on Combinatorial Optimization (EGRES), Eőtvős Loránd University, Hungary. (See website http://lemon.cs.elte.hu/egres/open/)

[7] A. Fraenkel, Planar kernel and Grundy with $d \leq 3, d_{\text {out }} \leq 2, d_{\text {in }} \leq 2$ are NP-complete, Discrete Appl. Math. 3 (1981), 257-262.

[8] J. Edmonds, S. Gaubert and V. Gurvich, Sperner oiks, Electron. Notes Discrete Math. 36 (2010), 1273-1280.

[9] H. Galeana-Sánchez and V. Neumann-Lara, Extending kernel perfect digraphs to kernel perfect critical digraphs, Discrete Math. 94 (1991), 181-187.

[10] M. Garey and D. Johnson, Computers and Intractability: A Guide to the Theory of NPCompleteness, Freeman and Co., New York, 1979.

[11] T. Király and J. Pap, Total dual integrality of Rothblum's description of the stable-marriage polyhedron, Math. Oper. Res. 33 (2008), 283-290.

[12] T. Király and J. Pap, A note on kernels and Sperner's lemma, Discrete Appl. Math. 157 (2009), 3327-3331.

[13] R. Irving, An efficient algorithm for the stable roommates problems, J. Algorithms 6 (1985), 577-595.

[14] D. Lichtenstein, Planar formulae and their uses, SIAM J. Comput. 11 (1982), 329-343.

[15] M. Richardson, Solutions of irreflexive relations, Ann. of Math. (2) 58 (1953), 573-590.

[16] U. Rothblum, Characterization of stable matchings as extreme points of a polytope, Math. Programming 54 (1992), 57-67.

[17] A. Schrijver, Theory of Linear and Integer Programming, John Wiley \& Sons, New York, 1986.

[18] J. von Neumann and O. Morgenstern, Theory of Games and Economic Behavior, Princeton University Press, Princeton, NJ, 1944. 